ARTICLE

\title{
Hyperproduction of 3-hydroxypropionate by Halomonas bluephagenesis
}

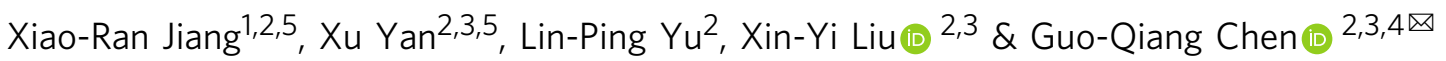

3-Hydroxypropionic acid (3HP), an important three carbon (C3) chemical, is designated as one of the top platform chemicals with an urgent need for improved industrial production. Halomonas bluephagenesis shows the potential as a chassis for competitive bioproduction of various chemicals due to its ability to grow under an open, unsterile and continuous process. Here, we report the strategy for producing 3HP and its copolymer poly(3-hydroxybutyrateco-3-hydroxypropionate) (P3HB3HP) by the development of $\mathrm{H}$. bluephagenesis. The transcriptome analysis reveals its $3 \mathrm{HP}$ degradation and synthesis pathways involving endogenous synthetic enzymes from 1,3-propanediol. Combing the optimized expression of aldehyde dehydrogenase $\left(\mathrm{AldD}_{\mathrm{Hb}}\right)$, an engineered $\mathrm{H}$. bluephagenesis strain of whose $3 \mathrm{HP}$ degradation pathway is deleted and that overexpresses alcohol dehydrogenases (AdhP) on its genome under a balanced redox state, is constructed with an enhanced 1.3-propanediol-dependent $3 \mathrm{HP}$ biosynthetic pathway to produce $154 \mathrm{~g} \mathrm{~L}^{-1}$ of $3 \mathrm{HP}$ with a yield and productivity of $0.93 \mathrm{~g} \mathrm{~g}^{-1} 1,3$-propanediol and $2.4 \mathrm{~g} \mathrm{~L}^{-1} \mathrm{~h}^{-1}$, respectively. Moreover, the strain could also accumulate $60 \%$ poly(3-hydroxybutyrate-co-32-45\% 3-hydroxypropionate) in the dry cell mass, demonstrating to be a suitable chassis for hyperproduction of 3HP and P3HB3HP.

\footnotetext{
${ }^{1}$ Department of Microbiology, Army Medical University, Chongqing, China. ${ }^{2}$ School of Life Sciences, Tsinghua University, Beijing, China. ${ }^{3}$ Center for Synthetic and Systems Biology, School of Life Sciences, Tsinghua University, Beijing, China. ${ }^{4}$ MOE Key Lab for Industrial Biocatalysis, Department of Chemical Engineering, Tsinghua University, Beijing, China. ${ }^{5}$ These authors contributed equally: Xiao-Ran Jiang, Xu Yan. ${ }^{凶}$ email: chengq@mail.tsinghua.edu.cn
} 
3 the -Hydroxypropionic acid (3HP) is a platform chemical for the synthesis of $\mathrm{C} 3$-based chemicals, including acrylic acid, malonic acid, acrylamide, acrylonitrile (ACN), and poly(3-hydroxypropionate) (P3HP) based polymers ${ }^{1-4}$. The chemical synthesis of $3 \mathrm{HP}$ is not suitable for industrial production owing to the high cost of the precursors and processes, and environmental incompatibility ${ }^{5}$. Since biosynthesis is sustainable and environmental friendly, it is considered a better choice for improved 3HP industrial production. Synthetic pathways were designed for producing $3 \mathrm{HP}$ from bio-renewable sources such as glucose, glycerol, or $\mathrm{CO}_{2}{ }^{6-9}$. However, its biosynthesis has been reported to have poor productivity ${ }^{6}$. Glucose utilizing pathways based on malonyl-CoA or $\beta$-alanine has been constructed for 3HP production ${ }^{10-12}$, but the complexity of manipulating and regulating multiple genes has proven to be a challenge for enhanced $3 \mathrm{HP}$ production ${ }^{13}$. Instead, glycerol, a byproduct of biodiesel, is more favorable. The glycerol to 3HP pathway involves glycerol dehydratase (GDHt) and aldehyde dehydrogenase (ALDH), converting glycerol to 3-hydroxypropionaldehyde and then 3HP, respectively ${ }^{14,15}$.

Traditional microbial chassis such as Escherichia coli or Corynebacterium glutamicum were reported to have the final $3 \mathrm{HP}$ concentration of approximately $70 \mathrm{~g} \mathrm{~L}^{-116,17}$. Klebsiella pneumoniae, which naturally produces vitamin $\mathrm{B}_{12}$ as a cofactor of GDHt, showed the highest production of $82 \mathrm{~g} \mathrm{~L}^{-1} 3 \mathrm{HP}^{18-20}$. However, a larger-scale production using $K$. pneumoniae was impossible because it is been known as an opportunity pathogen ${ }^{13}$. Therefore, to enhance the production of $3 \mathrm{HP}$, we need to develop a more suitable producer as the chassis. Another issue occurs during the production of 3HP: under an aerobic condition, GDHt is deactivated combined with a downregulated expression of vitamin $\mathrm{B}_{12}{ }^{21}$, while under anaerobic condition, cells were grown poorly, and $\mathrm{NAD}^{+}$, a necessary cofactor of ALDH, has difficulty to regenerate ${ }^{21-24}$. The trade-off between vitamin $\mathrm{B}_{12}$ synthesis and $\mathrm{NAD}^{+}$regeneration controls 3HP production and the key is the balance of dissolved oxygen in the cultures ${ }^{23}$. Pure 1,3-propanediol (PDO), obtainable from microbial process $\left(1 \$ \mathrm{~kg}^{-1}\right.$ on www.alibaba.com, unpurified 1,3-propanediol should have a much lower price), is a monomer of a high-performed textile material, poly(1,3-trimethylene terephthalate) (PTT) ${ }^{25}$. Using 1.3-propanediol as the substrate may be a possible solution ${ }^{26-28}$, as 1,3-propanediol can also be converted into 3HP using 1,3-propanediol oxidoreductase (PDOR) and $\mathrm{ALDH}^{27}$. The two $\mathrm{NAD}^{+}$-dependent enzymes PDOR and ALDH function well under aerobic conditions without vitamin $\mathrm{B}_{12}{ }^{21}$. As an example, Gluconobacter oxydans was reported to produce $45 \mathrm{~g} \mathrm{~L}^{-1}$ $3 \mathrm{HP}$ using these two enzymes ${ }^{27}$. These results paved the way by using 1,3-propanediol as the carbon source to produce 3HP.

Halophilic Halomonas spp. have attracted attentions from industrial biotechnology researchers ${ }^{29}$. They are capable of growing at high concentrations of $\mathrm{NaCl}$ and alkaline $\mathrm{pH}$, thus, avoiding possible contamination by other microorganisms even under open unsterile conditions ${ }^{30}$. As a result, Halomonas spp.-based fermentations have reduced cost and complexity ${ }^{31,32}$. Pilot-scale fermentations using seawater-based artificial media under open and continuous conditions were conducted without contamination over a long period of two months ${ }^{32}$. Moreover, several synthetic biology and chromosome engineering methods such as CRISPR/ Cas9 have been successfully applied in Halomonas spp., turning them into a more suitable chassis for future metabolic engineering and genome editing ${ }^{33,34}$. Next generation of industrial biotechnology (NGIB) has been developed based on extremophile Halomonas spp., allowing the production to be conducted under open, unsterile, and continuous process conditions with reduced fresh water and energy consumption, aiming for increasing economic competitiveness of current industrial biotechnology 29,35,36.
Halomonas bluephagenesis TD (once termed Halomonas sp. TD01) is a halophile isolated from Aydingol Lake in Xinjiang Province/China, has been used as a chassis for the production of several bioplastic polyhydroxyalkanoates (PHAs), including poly-3-hydroxybutyrate (PHB), poly(3-hydroxybutyrate-co-4hydroxybutyrate) (P3HB4HB) and poly(3-hydroxybutyrate-co3-hydroxyvalerate) (PHBV) using various engineering approaches ${ }^{37-40}$. H. bluephagenesis is capable of growing at $\mathrm{NaCl}$ concentrations of $20-150 \mathrm{~g} \mathrm{~L}^{-1}$ with an optimal concentration of $40-60 \mathrm{~g} \mathrm{~L}^{-1}$. The strain can be grown at a $\mathrm{pH}$ ranging 5.0-11.0, the optimum of which is 8.5-9.0. However, as a promising biosynthetic chassis, $H$. bluephagenesis should further expand its product diversity to create more industrial value $^{41}$. The unique growth condition of high osmotic pressure for $H$. bluephagenesis is beneficial for producing organic acid at a high concentration ${ }^{35}$. The organic acids produced are in the form of sodium salt under alkaline culture conditions, preventing the formation of toxic undissociated organic acids during production ${ }^{42,43}$. It is therefore reasonable to expect that H. bluephagenesis can be a suitable organic acid producer such as 3-hydroxypropionic acid (3HP).

Here, we show that a coherent bioprocess based on halophilic H. bluephagenesis for production of $3 \mathrm{HP}$ and its copolymer poly (3-hydroxybutyrate-co-3-hydroxypropionate) (P3HB3HP) by identifying and deleting $3 \mathrm{HP}$ degradation pathway, screening and optimizing endogenous biosynthetic enzymes, engineering genomic expression of 3HP synthesis pathway and balancing redox states. The engineered $H$. bluephagenesis strain produces a higher titer of $3 \mathrm{HP}$ with 1,3-propanediol as a carbon source under open and unsterile fed-batch fermentation.

\section{Results}

Identification of 3HP degradation pathway in $\mathrm{H}$. bluephagenesis. Due to the high osmotic pressure tolerance, $H$. bluephagenesis is an ideal chassis for the extracellular production of small molecules with a high concentration in the cultures. The extracellular $3 \mathrm{HP}$ tolerance has been demonstrated by $H$. bluephagenesis, further proving its potential as a microbial industrial chassis for $3 \mathrm{HP}$ (Supplementary Fig. 1). To verify the feasibility of 3HP production by $H$. bluephagenesis, genes dha $T_{P p}$ and ald $D_{P p}$ from Pseudomonas putida KT2440 encoding 1,3-propanediol dehydrogenase and aldehyde dehydrogenase, were constructed in plasmid p30 and overexpressed in E. coli S17-1 and H. bluephagenesis, respectively (Fig. 1a). The recombinant E. coli S17-1 (p30) produced $1.5 \mathrm{~g} \mathrm{~L}^{-1}$ and $H$. bluephagenesis (p30) produced $0.2 \mathrm{~g} \mathrm{~L}^{-1} 3 \mathrm{HP}$ when grown in conical flasks. The former strain was cultured in LB medium supplemented with $30 \mathrm{~g} \mathrm{~L}^{-1}$ glucose and $10 \mathrm{~g} \mathrm{~L}^{-1}$ 1,3-propanediol, and the latter was grown in the defined minimal medium supplemented with $30 \mathrm{~g} \mathrm{~L}^{-1}$ glucose and $10 \mathrm{~g} \mathrm{~L}^{-1}$ 1,3-propanediol (Fig. 1b). Recombinant H. bluephagenesis (p30) accumulated $0.5 \mathrm{~g} \mathrm{~L}^{-1} \mathrm{PHB}$ when $5 \mathrm{~g} \mathrm{~L}^{-1} 3 \mathrm{HP}$ was added as the sole carbon source (Fig. 1c). The 3HP may be degraded to acetyl-coA for cell growth and PHB accumulation, indicating that $H$. bluephagenesis is able to utilize $3 \mathrm{HP}$ as a carbon source (Fig. 1a). This result suggests that $H$. bluephagenesis contains a 3HP degradation pathway.

To investigate the key genes in the 3HP degradation pathway, transcriptome analysis of $H$. bluephagenesis was performed using the cells grown under culture conditions with and without $3 \mathrm{HP}$ as the carbon source, respectively. A volcano plot illustrates the distribution of differentially expressed genes (DEGs) (Fig. 1d). Overall, when $3 \mathrm{HP}$ was used as the sole carbon source for growth, 384 genes in the cells were upregulated while 210 were downregulated compared with glucose utilized as a sole carbon source (Fig. 1d and Supplementary Data 2). Possibly similar to the catabolic operons, the addition of $3 \mathrm{HP}$ could activate its 
a

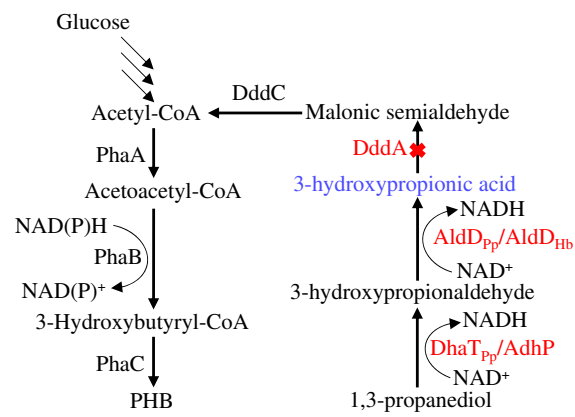

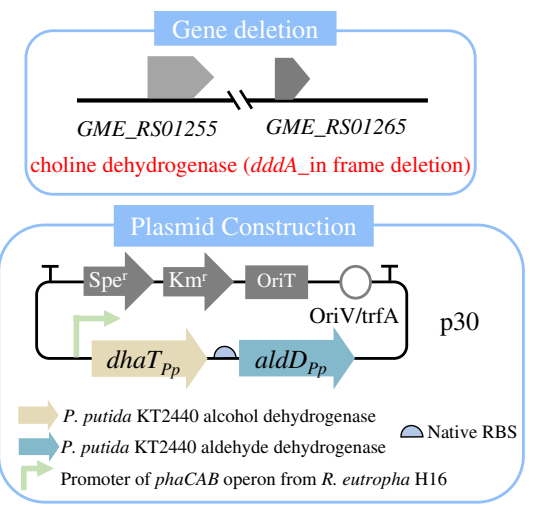

Promoter of phaCAB operon from R. eutropha $\mathrm{H} 16$

d
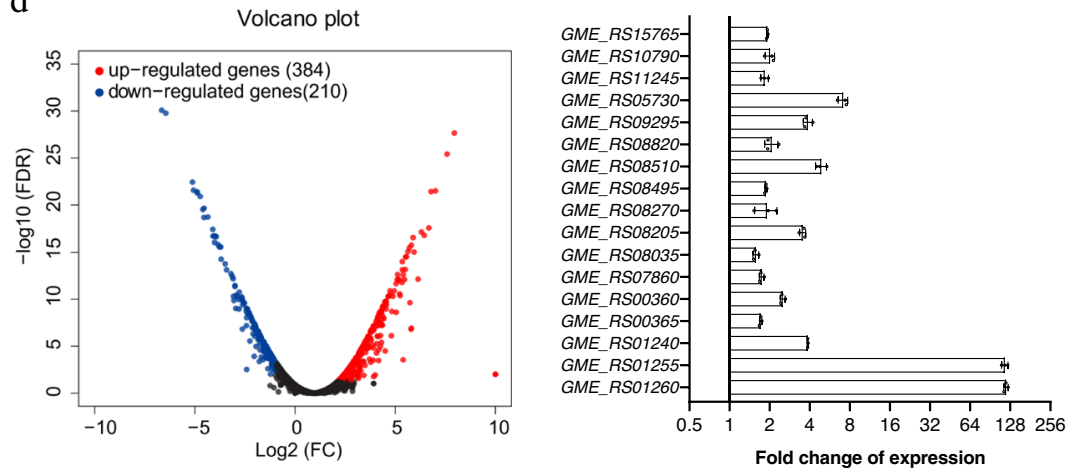

b
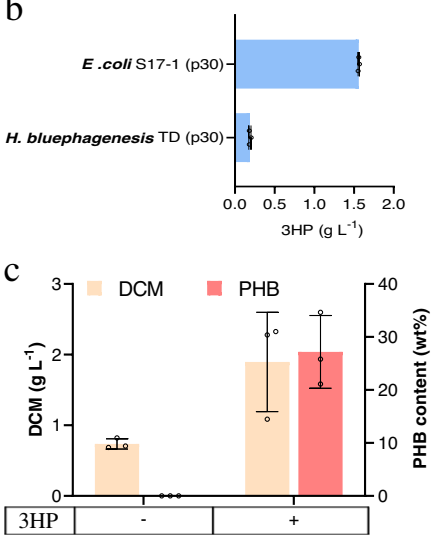

e

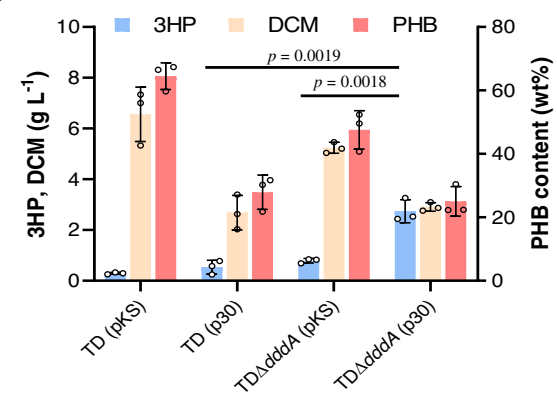

Fig. 1 Metabolic engineering of $\boldsymbol{H}$. bluephagenesis for 3HP production. a Overall strategies for $3 \mathrm{HP}$ production involving a combination of marker-free $d d d A$ deletion and plasmid-expressing $d h a T_{P p}$, ald $D_{P p}$. Red color " $X$ " indicates inactivation of metabolic pathways. Gene dhaT $T_{P p}$ encodes $P$. putida KT2440 alcohol dehydrogenase, $a l d D_{P p}$ encodes $P$. putida $\mathrm{KT} 2440$ aldehyde dehydrogenase, adhP encodes $H$. bluephagenesis alcohol dehydrogenase, ald $D_{H b}$ encodes $H$. bluephagenesis aldehyde dehydrogenase. DddA putative 3-hydroxypropionate dehydrogenase in $\mathrm{H}$. bluephagenesis, DddC CoA-acylating methylmalonate-

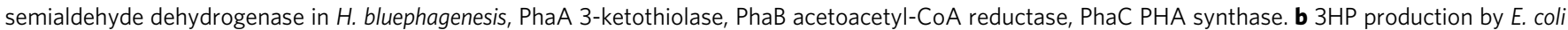
and $H$. bluephagenesis harboring plasmid p30, respectively. c PHB production by $H$. bluephagenesis cultured on $5 \mathrm{~g} \mathrm{~L}^{-1} 3 \mathrm{HP}$ as a sole carbon source. d Influence of 3HP on cell metabolism at the transcriptional level in $\mathrm{H}$. bluephagenesis. Volcano plot and fold change of gene expression of differentially expressed gene (DEG) distribution. A corrected P-value of 0.05 and log2 (fold change) of 1 were set as the thresholds for significantly differential expression. Red, upregulated genes; blue, downregulated genes. qRT-PCR analysis of several putative upregulated dehydrogenases expression levels in the $3 \mathrm{HP}$ containing cultures compared to that of the glucose-containing ones. e $3 \mathrm{HP}$ and PHB production by $H$. bluephagenesis TD $\Delta d d d A$ harboring plasmid p30. Cells were grown in a defined minimal medium supplemented with $30 \mathrm{~g} \mathrm{~L}^{-1}$ glucose and $10 \mathrm{~g} \mathrm{~L} \mathrm{~L}^{-1} 1,3$-propanediol. All titers were obtained after $48 \mathrm{~h}$ cultivation at 200 r.p.m. at $37^{\circ} \mathrm{C}$. The initial pH of all shake-flask studies was 9. All data represent the mean of $n=3$ biologically independent samples and error bars show s.d. Two-tailed Student's $t$-tests were performed to determine the statistical significance for two-group comparisons. DCM dry cell mass, PHB polyhydroxybutyrate.

degradation pathway. Several putative upregulated dehydrogenases were thus selected for qRT-PCR verification. Among them, GME_RS01260 encoding choline dehydrogenase (RefSeq. Accession: WP_009721523) and GME_RS01255 encoding CoAacylating methylmalonate-semialdehyde dehydrogenase (RefSeq. Accession WP_009721522), were upregulated by 119-folds and 116-folds, respectively (Fig. 1d). Choline dehydrogenase is named DddA due to its high homology with the DddA of Halomonas sp. HTNK1 $^{44}$ (Fig. 1a). Deletions of putative 3-hydroxypropionate dehydrogenase $(\mathrm{HpdH})$ and putative 3-hydroxyisobutyrate dehydrogenase $(\mathrm{HbdH}-4)$ in Pseudomonas denitrificans eliminate its ability to degrade $3 \mathrm{HP}^{45-47}$. Cell growth was found to be unaffected while $3 \mathrm{HP}$ could no longer be utilized as the sole carbon source after the $d d d A$ deletion (Supplementary Figs. 2 and 3 ). The $d d d A$ gene deletion in recombinant $H$. bluephagenesis $\mathrm{TD} \Delta d d d A$ (p30) could increase the production with $2.7 \mathrm{~g} \mathrm{~L}^{-1}$ $3 \mathrm{HP}$ and $0.7 \mathrm{~g} \mathrm{~L}^{-1} \mathrm{PHB}$ when grown in conical flasks (Fig. 1e).

The toxicity of 3-hydroxypropionaldehyde (3HPA), an intermediate for $3 \mathrm{HP}$ biosynthesis, negatively affects cell growth and $3 \mathrm{HP}$ production. Expression modules were designed to prevent the possible intracellular accumulation of 3HPA using optimized promoter, ribosome binding site (RBS) sequence of $a l d D_{P p}$, and shifting the order of ald $D_{P p}$ and $d h a T_{P p}$ operon (Supplementary Fig. 4a). Plasmid p31 was constructed replacing the original RBS with a stronger one; $\mathrm{p} 32$ contains changing order of ald $D_{P p}$ and $d h a T_{P p}$ operon; $\mathrm{p} 33$ and $\mathrm{p} 34$ based on $\mathrm{p} 32$ were constructed with a stronger RBS and promoter, respectively.

qRT-PCR was performed to further confirm the enhanced expression level of $a l d D_{P p}$ gene under the $\mathrm{P}_{\text {Porin }}$ promoter ${ }^{48}$. Recombinant strains were cultured in conical flasks and grown for $6,12,24,36$, and $48 \mathrm{~h}$, respectively. The results showed that the expression level of ald $D_{P p}$ in $H$. bluephagenesis TD $\Delta d d d A$ harboring $\mathrm{p} 34$ was higher compared to that of $H$. bluephagenesis TD $\Delta d d d A$ (p32) during the early growth phase, whereas an opposite trend was revealed at the late growth period (Supplementary Fig. $4 \mathrm{~b}$ ). However, the enzyme activity of $\mathrm{AldD}_{\mathrm{Pp}}$ was observed to decrease over time, while the activity of DhaT $\mathrm{T}_{\mathrm{Pp}}$ has shown an increase up to 24 hours (Supplementary Fig. 5). Additionally, we compared the enzyme activity of $\mathrm{AldD}_{\mathrm{Pp}}$ in recombinant $H$. bluephagenesis TD $\Delta d d d A$ harboring p32-p34 deleted with $d h a T_{P p}$ gene. The activity of $A_{1 d D_{P p}}$ was increased using the optimized promoter or ribosome binding site (RBS) sequence of ald $D_{P p}$, even though the yield of $3 \mathrm{HP}$ did not increase significantly (Supplementary Fig. 6a, b). In general, manipulation 
of ald $D_{P p}$ expression level showed no enhancement on 3HP production (Supplementary Fig. 6a). This may be due to the low activity of $\mathrm{DhaT}_{\mathrm{Pp}}$, which leads to the weak production of toxic 3HPA intermediate. Hence, even though the poor expression of ald $D_{P p}$, it was enough for consuming the produced 3HPA and avoiding its accumulation. It was also shown that the specific activity of $\mathrm{DhaT}_{\mathrm{Pp}}$ was lower than that of 1,3-propanediol oxidoreductase $\left(\mathrm{DhaT}_{\mathrm{Kp}}\right.$ ) in Klebsiella pneumoniae DSM 2026 as reported $^{49}$ (Supplementary Fig. 6c), indicating an insufficient supply of 3HPA, which limits $3 \mathrm{HP}$ biosynthesis. To develop microbial cell factories for efficient $3 \mathrm{HP}$ production, efficient enzyme systems must be constructed ${ }^{50}$.

Analysis of endogenous enzymes converting 1,3-propanediol to 3HP. A trace amount of $3 \mathrm{HP}$ was detected in the $H$. bluephagenesis TD $\Delta d d d A$ harboring the empty plasmid as a control, indicating the existence of endogenous activity of native $3 \mathrm{HP}$ synthetic enzymes in $H$. bluephagenesis (Fig. 1e). To analyze the endogenous synthesis pathway of $3 \mathrm{HP}$, a protein homologous to the aldehyde dehydrogenase $\operatorname{AldD}_{\mathrm{Pp}}$ was found via in silico analysis of the genome sequence of $H$. bluephagenesis, namely, $G M E \_R S 00360$, which was annotated as the putative ald $D_{H b}$ gene encoding a 506-amino-acid polypeptide. An overall $80 \%$ amino acid identity was shown between $\mathrm{AldD}_{\mathrm{Hb}}$ (RefSeq. Accession: WP_009721344) and the $\mathrm{AldD}_{\mathrm{Pp}}$ from P. putida KT2440 after BLAST analysis.

Subsequently, an transcriptome analysis of $H$. bluephagenesis was performed using cells grown with or without 1,3-propanediol in culture media containing $30 \mathrm{~g} \mathrm{~L}^{-1}$ glucose to investigate alcohol dehydrogenases in $H$. bluephagenesis. The volcano plot illustrates the distribution of differentially expressed genes (DEGs) (Fig. 2a). Overall, 292 genes were upregulated while 285 were downregulated in the presence of 1,3-propanediol in the cultures (Fig. 2a and Supplementary Data 3). Several putative upregulated alcohol dehydrogenases were examined, including $G M E \_R S 01345, G M E \_05160, G M E \_R S 01585$ and GME_RS00365 encoding NAD(P)-dependent alcohol dehydrogenase (RefSeq. Accession: WP_009721539.1), zinc-binding alcohol dehydrogenase (GenBank Accession: EGP20677.1), zinc-binding dehydrogenase (RefSeq. Accession: WP_009721587.1), and alcohol dehydrogenase AdhP (RefSeq. Accession: WP_039868491.1), respectively (Fig. 2b).

In order to overexpress the mentioned genes encoding putative alcohol dehydrogenases, four related plasmids were constructed combined with the gene $a l d D_{H b}$ encoding aldehyde dehydrogenase, and $H$. bluephagenesis TD $\Delta d d d A$ was transformed using the resulting plasmids, respectively (Fig. 2b). The result showed only one strain harboring GME_RS00365 gene adhP was able to produce $3.3 \mathrm{~g} \mathrm{~L}^{-1} 3 \mathrm{HP}$ (Fig. 2b). Thus, the gene adhP was identified to encode the 1,3-propanediol dehydrogenase with relatively high activity to convert 1,3propanediol to $3 \mathrm{HP}$.

An expression module, which does not hamper the cell growth during the $3 \mathrm{HP}$ production, was designed by optimizing the expression levels of ald $D_{H b}$ encoding aldehyde dehydrogenase (Fig. 2c). The $H$. bluephagenesis TD $\Delta d d d A$ harboring p59 equipped with a stronger RBS and p60 with a stronger promoter, respectively, achieved a higher yield of $\sim 7 \mathrm{~g} \mathrm{~L}^{-1} 3 \mathrm{HP}$, compared to the control $H$. bluephagenesis TD $\Delta d d d A$ harboring $\mathrm{p} 58$ without a stronger promoter or RBS (Fig. 2c). Moreover, polyhydroxybutyrate (PHB) contents produced by $H$. bluephagenesis TD $\Delta d d d A$ (p58), H. bluephagenesis TD $\Delta d d d A$ (p59) and $H$. bluephagenesis TD $\Delta d d d A$ (p60) were similar (Fig. $2 \mathrm{~d}$ ). The above results demonstrated that the ald $D_{H b}$ upregulated expression level could enhance $3 \mathrm{HP}$ production. In addition, we compared the enzyme activity of $\operatorname{AldD}_{\mathrm{Hb}}$ in the recombinant $H$. bluephagenesis TD $\Delta d d d A$ harboring p58, p59 or p60 deleted with the adhP gene (Fig. 2e). Consistent with the significant increase of $3 \mathrm{HP}$ yield, the enzyme activity of $\mathrm{AldD}_{\mathrm{Hb}}$ was significantly increased by optimizing the promoter or ribosome binding site (RBS) sequence of ald $D_{P p}$ (Fig. 2e). Since 1,3-propanediol was almost completely consumed while glucose was still available at $48 \mathrm{~h}$ of cultivation when $30 \mathrm{~g} \mathrm{~L}^{-1}$ glucose and $10 \mathrm{~g} \mathrm{~L}^{-1} 1,3$ propanediol were added to cultures, a higher final 3HP titer is expected when there are more 1,3-propanediol and less glucose (Supplementary Fig. 7).

Selection of efficient enzymes for biosynthesis of 3HP in $H$. bluephagenesis. With the successful establishment of $3 \mathrm{HP}$ production platform based on $H$. bluephagenesis, 3HP production needed further improvement. The $3 \mathrm{HP}$ biosynthetic pathway from 1,3-propanediol comprises two parts, including the formation of 3HPA and subsequent conversion of $3 \mathrm{HPA}$ to $3 \mathrm{HP}$ (Fig. 1a). To select the efficient enzyme combinations for the $3 \mathrm{HP}$ production via 1,3-propanediol oxidoreductase and aldehyde dehydrogenase, different plasmids were constructed to express variants of different pathway components. Specifically, aldehyde dehydrogenase AldH (RefSeq. Accession: WP_001009090.1) of $E$. coli MG1655, 1,3-propanediol oxidoreductase DhaT $\mathrm{Kp}_{\mathrm{Kp}}$ (GenBank AAP97875.1) and aldehyde dehydrogenase PuuC (RefSeq. Accession: WP_004224052.1) of K. pneumoniae were selected (Fig. 3a) ${ }^{49,51,52}$. In the enzyme-screening process, we first compared the efficiency of the selected 1,3-propanediol oxidoreductase encoding genes in combination with ald $D_{H b}$ (plasmids p90 and p95). With the co-overexpression of $a d h P$, the resultant strain $H$. bluephagenesis TD $\Delta d d d A$ (p90) was shown to have the highest 3HP production (Fig. 3b). Subsequently, the selected aldehyde dehydrogenases encoding genes were evaluated based on adhP (plasmids p91, p99, and p100). The aldD ${ }_{H b}$ still outperformed its counterparts, producing $3 \mathrm{HP}$ at a titer of $7.8 \mathrm{~g} \mathrm{~L}^{-1}$ (H. bluephagenesis TD $\Delta d d d A$ (p91), Fig. 3c). However, H. bluephagenesis TD $\Delta d d d A$ (p105) containing the K. pneumoniae PuuC combination with $K$. pneumoniae $\mathrm{DhaT}_{\mathrm{Kp}}$ only generated $3 \mathrm{HP}$ at a titer of $2 \mathrm{~g} \mathrm{~L}^{-1}$ (Supplementary Fig. 8a).

Enzyme activities in the recombinant $H$. bluephagenesis TD $\Delta d d d A$ overexpressing the three different alcohol dehydrogenases (AdhP, DhaT $\mathrm{Pp}_{\mathrm{Pp}}$, and $\mathrm{DhaT}_{\mathrm{Kp}}$ ) and four different aldehyde dehydrogenases $\left(\mathrm{AldD}_{\mathrm{Hb}}, \mathrm{AldD}_{\mathrm{Pp}}, \mathrm{AldH}\right.$, and PuuC) were analyzed using their corresponding purified enzymes, respectively (Fig. 3a). The His-tagged enzymes were purified to electrophoretic homogeneity (Supplementary Fig. 9). For the oxidative activity, the specific activity of AdhP was found to be $\sim 7.0 \mathrm{U} \mathrm{mg}^{-1}$, higher than the alcohol dehydrogenase of $P$. putida DhaT $_{\mathrm{Pp}}$ and K. pneumoniae DhaT $\mathrm{Kp}$ (Fig. 3d). The aldehyde dehydrogenase enzyme is in general presented as a tetramer. Its $\mathrm{C}$ terminus has been known to play an important role in the assembly, and thus a His-tag was introduced to the $\mathrm{N}$ terminus. However, the production of $3 \mathrm{HP}$ was significantly reduced when the His-tag was introduced to the $\mathrm{N}$ terminus of $\mathrm{AldD}_{\mathrm{Hb}}$ (Supplementary Fig. 8b). Thus, we introduced the His-tag to the $\mathrm{C}$ terminus of $\mathrm{AldD}_{\mathrm{Hb}}$. The specific activity of $\mathrm{AldD}_{\mathrm{Hb}}$ was $\sim 3.7 \mathrm{U} \mathrm{mg}^{-1}$, the highest among all the aldehyde dehydrogenases (Fig. 3e). The AdhP and $\mathrm{AldD}_{\mathrm{Hb}}$ enzymes kinetic properties of $K_{\text {cat }}$ and $K_{\mathrm{m}}$ were characterized (Table 1). The half-saturation constant $\left(K_{\mathrm{m}}\right)$ of $\mathrm{AldD}_{\mathrm{Hb}}$ was low at $0.05 \mathrm{mM}$, indicating that the $\mathrm{AldD}_{\mathrm{Hb}}$ has a very high affinity for the substrate $3 \mathrm{HPA}$, and the catalytic efficiency $\left(k_{\text {cat }} / K_{\mathrm{m}}\right)$ of the $\mathrm{AldD}_{\mathrm{Hb}}$ was 100 times higher than that of PuuC at the same condition (Supplementary Table 3 ). Thus, $\mathrm{AldD}_{\mathrm{Hb}}$ and $\mathrm{AdhP}$ were considered as the efficient enzymes for biosynthesis of $3 \mathrm{HP}$ by $H$. bluephagenesis. 
a

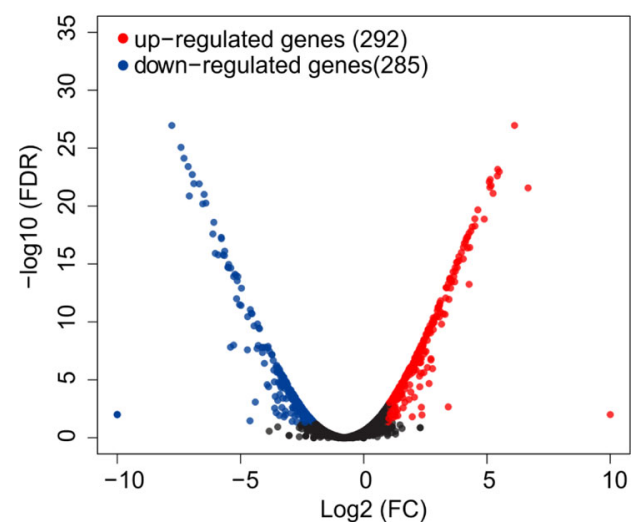

$\mathrm{c}$

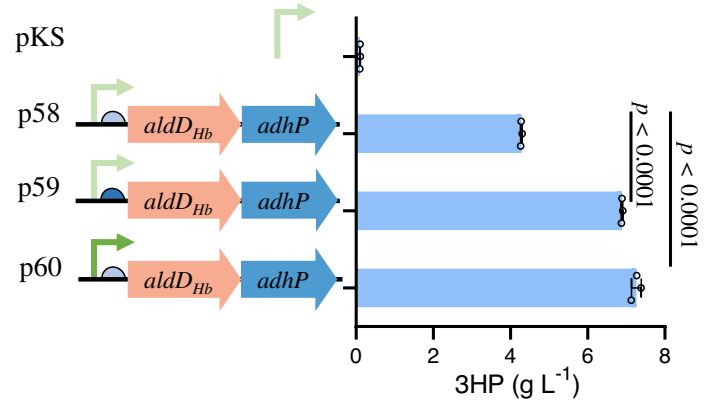

b

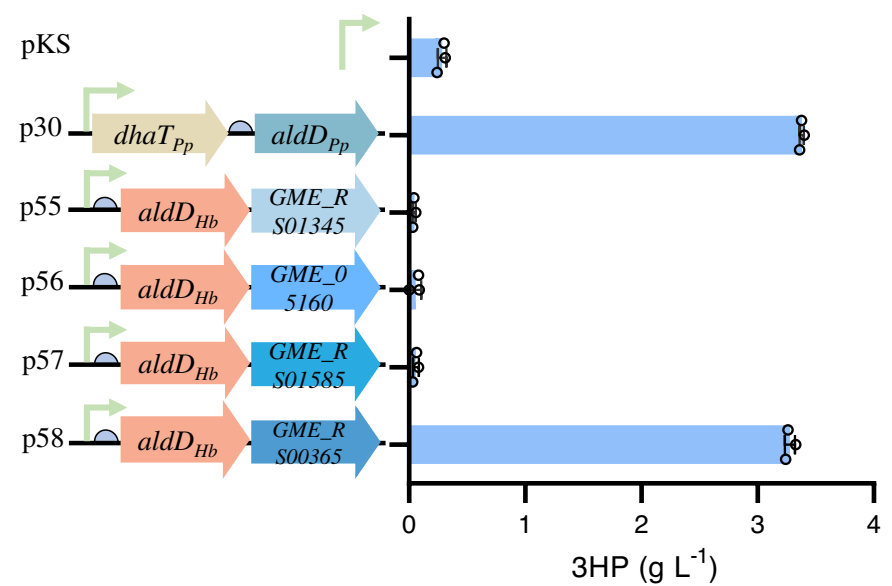

d

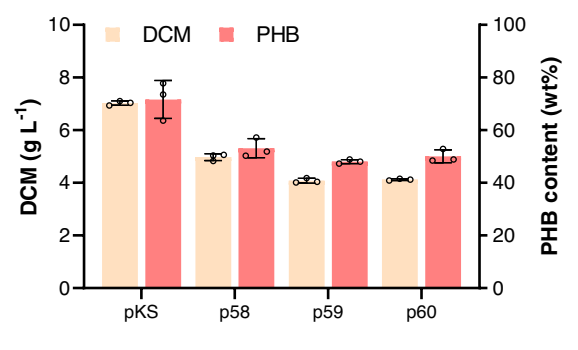

e

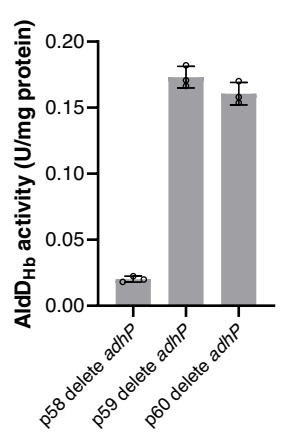

Fig. 2 Selection of endogenous enzymes catalyzing 1,3-propanediol to 3HP biosynthesis. a Influence of 1,3-propanediol on cell metabolism at the transcriptional level in $H$. bluephagenesis TD $\Delta d d d A$. Volcano plot and fold change of gene expression of differentially expressed gene (DEG) distribution. A corrected $P$-value of 0.05 and log2 (fold change) of 1 were set as the thresholds for significantly differential expression. Red, upregulated genes; blue, downregulated genes. b $3 \mathrm{HP}$ production by $\mathrm{H}$. bluephagenesis TD $\Delta d d d A$ overexpressing different biosynthetic genes using 1,3-propanediol as a carbon source. Plasmid pKS and p30 were used as the negative and positive controls, respectively. Gene dhaT $T_{P p}$ encodes $P$. putida KT2440 alcohol dehydrogenase, ald $D_{P p}$ encodes $P$. putida KT2440 aldehyde dehydrogenase, ald $D_{H b}$ encodes $H$. bluephagenesis aldehyde dehydrogenase, GME_RSO1345 encodes NAD(P)dependent alcohol dehydrogenase; GME_05160 encodes zinc-binding alcohol dehydrogenase, GME_RSO1585 encodes zinc-binding dehydrogenase, GME_RS00365 encodes alcohol dehydrogenase AdhP. c 3HP production and $\mathbf{d}$ cell growth (DCM) and PHB content under optimal expression levels of aldehyde dehydrogenase. A stronger RBS was cloned instead of the original construct p58 to form plasmid p59. A stronger promoter Pporin replaced the original construct $\mathrm{p} 58$ to generate plasmid p60 (Supplementary Table 5). Cells were grown in the defined minimal medium supplemented with $30 \mathrm{~g} \mathrm{~L}^{-1}$ glucose, $10 \mathrm{~g} \mathrm{~L}^{-1} 1$,3-propanediol, and $3 \mathrm{~g} \mathrm{~L}^{-1}$ acetic acid. All titers were obtained after $48 \mathrm{~h}$ cultivation at $200 \mathrm{r}$.p.m. and $37^{\circ} \mathrm{C}$. Initial pH of all shake-flask studies was 9. Two-tailed Student's $t$-tests were performed to determine the statistical significance for two-group comparisons. e The enzyme activity of AldD $_{\mathrm{Hb}}$ was assayed using the recombinant $H$. bluephagenesis TD $\Delta d d d A$ harboring p58-p60 deleted with adhP gene cultivated in 60-LB medium for $24 \mathrm{~h}$. The comparison of activities was conducted using a crude extract. All data represent the mean of $n=3$ biologically independent samples and error bars show s.d.

Combinatorial optimization for increasing the production of 3HP. To stabilize microbial production, it is critical to obtain stable gene expressions in $H$. bluephagenesis. This is commonly accomplished via genomic integration of genes of interest. To efficiently manipulate relevant genes, a marker-free CRISPR/Cas9 genome-editing technology was employed ${ }^{33}$, allowing the construction of a $3 \mathrm{HP}$ biosynthetic pathway for genome integration (Fig. 4a). The best-performing gene combination, ald $D_{H b}$ with adhP containing promoter of phaCAB operon from Ralstonia eutropha $\mathrm{H} 16$ and a stronger RBS, were integrated into the chromosome of $H$. bluephagenesis for evaluation on $3 \mathrm{HP}$ production (Fig. 4a). The genomic integration of the 3HP biosynthetic pathway does not negatively impact cell growth (Supplementary Fig. 10). As a result, a significant increase of $3 \mathrm{HP}$ production was observed in recombinant $H$. bluephagenesis TD22 and $H$. bluephagenesis TD27 containing intact PHB production pathway when compared with its $\mathrm{PHB}$ synthesis operon phaCAB deleted strains $H$. bluephagenesis TD17 and H. bluephagenesis TD25, respectively, indicating that the PHB accumulation could promote 3HP synthesis. Moreover, H. bluephagenesis TD27 harboring genome-expressing 3HP endogenous synthetic pathway produced $\sim 9 \mathrm{~g} \mathrm{~L}^{-1} 3 \mathrm{HP}$, slightly higher than that synthesized by the plasmid-expressing $H$. bluephagenesis TD $\Delta d d d A$ (p59). Therefore, $H$. bluephagenesis TD27 was taken as the platform for further enhanced 3HP production from 1,3-propanediol (Fig. 4b).

$1 \mathrm{M} 3 \mathrm{HP}$ can be generated from $1 \mathrm{M}$ 1,3-propanediol, accompanied by the generation of $2 \mathrm{M} \mathrm{NADH}$ (Fig. 1a). However, an abnormal increase of $\mathrm{NADH}$ could lead to cell injury, reduced glycolysis, and TCA cycle ${ }^{53}$. It has been reported that surplus $\mathrm{NADH}$ can be used for the reductive metabolism of acetic acid to ethanol in some Clostridium species ${ }^{54}$. Acetate is a cost-effective carbon source, which can be obtained from carbon dioxide and hydrolysis or pyrolysis of lignocellulosic biomass like corn stover. Gradient concentrations of acetic acid $\left(0,3,6\right.$, and $\left.9 \mathrm{~g} \mathrm{~L}^{-1}\right)$ were cofed to the culture medium of $H$. bluephagenesis TD27 with $20 \mathrm{~g} \mathrm{~L}^{-1}$ glucose and $20 \mathrm{~g} \mathrm{~L}^{-1}$ 1,3-propanediol, respectively (Fig. 4c). The addition of $3 \mathrm{~g} \mathrm{~L}^{-1}$ acetic acid enhanced $3 \mathrm{HP}$ production to $8.6 \mathrm{~g} \mathrm{~L}^{-1}$ in shake-flask studies, representing a $35 \%$ increase compared to cultures in the absence of acetic acid. As a result, cell growth in terms of dry cell mass (DCM) and PHB content 
a

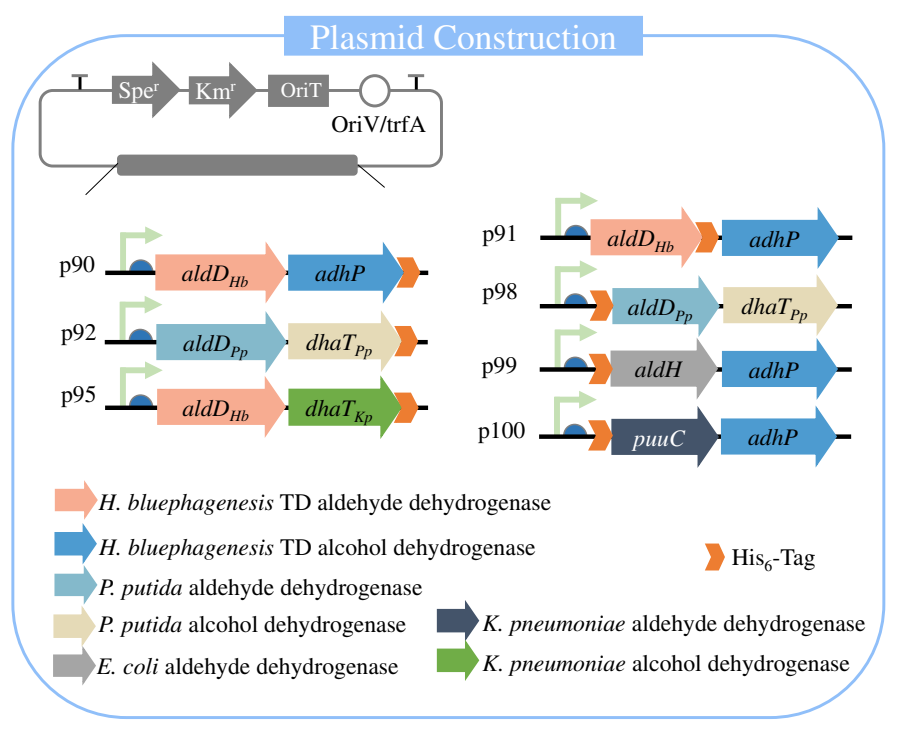

b

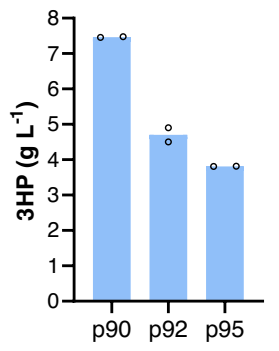

$\mathrm{d}$

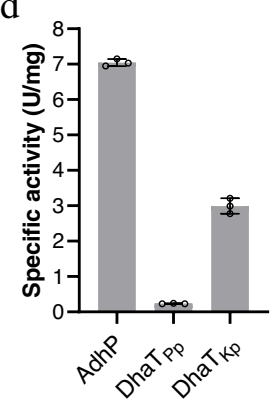

$\mathrm{C}$

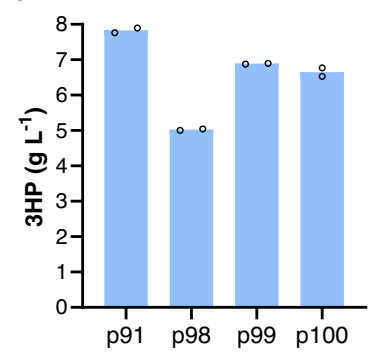

$\mathrm{e}$

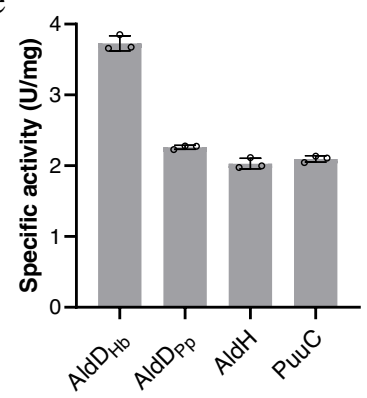

Fig. 3 Selection of efficient enzymes for biosynthesis of $\mathbf{3 H P}$ in $\boldsymbol{H}$. bluephagenesis. a Construction of the plasmids to express enzymes from different species. Following plasmids were constructed: plasmid p90 expressing adhP gene with a His-tag on C-terminal, p91 expressing aldD $D_{H b}$ gene with a His-tag on C-terminal, p92 expressing dhaT $T_{p p}$ gene with a His-tag on C-terminal and p98 expressing aldD $D_{P p}$ gene with a His-tag on $\mathrm{N}$-terminal. The aldH gene, with a His-tag on N-terminal, was amplified via PCR using E. coli genomic DNA as a template and cloned into the p58 by replacing the aldD $D_{H b}$ gene. This plasmid is labeled as p99. The $d h a T_{K p}$ or puuC gene, with a His-tag, was amplified via PCR using K. pneumoniae DSMZ 2026 genomic DNA as a template and cloned into the $\mathrm{p} 58$ by replacing the $a d h P$ or ald $D_{H b}$ gene. These plasmids were labeled as $\mathrm{p} 95$ and p100. 3HP production of different alcohol dehydrogenases (b), and different aldehyde dehydrogenases (c). Cells were grown in the defined minimal medium containing $20 \mathrm{~g} \mathrm{~L}^{-1} \mathrm{glucose}_{2} 20 \mathrm{~g} \mathrm{~L}^{-1} 1,3-\mathrm{propanediol}$ and $3 \mathrm{~g} \mathrm{~L}^{-1}$ acetic acid. All titers were obtained after $48 \mathrm{~h}$ cultivation at 200 r.p.m. and $37^{\circ} \mathrm{C}$. The initial pH of all shake-flask studies was 9 . All data represent the mean of $n=2$ biologically independent. The comparation of the various alcohol dehydrogenases oxidative activities (d), and various aldehyde dehydrogenases activities (e) in a purified enzyme. All data represent the mean of $n=3$ biologically independent samples and error bars show s.d.

Table 1 Kinetic parameters of $\mathrm{AdhP}_{\mathrm{d}}$ and $\mathrm{AldD}_{\mathrm{Hb}}$.

\begin{tabular}{|c|c|c|c|}
\hline Enzymes & $K_{m}(m M)$ & $K_{\text {cat }}\left(s^{-1}\right)$ & $\begin{array}{l}K_{\text {cat }} / K_{m} \\
\left(s^{-1} \mathbf{m M}^{-1}\right)\end{array}$ \\
\hline$\overline{A d h P a}$ & $35.41 \pm 1.525$ & $4.11 \pm 0.05$ & $0.12 \pm 0.004$ \\
\hline AdhPb & $1.75 \pm 0.278$ & $19.39 \pm 0.62$ & $11.19 \pm 1.304$ \\
\hline AldD $_{\mathrm{Hb}}{ }^{\mathrm{c}}$ & $0.05 \pm 0.002$ & $3.38 \pm 0.09$ & $67.45 \pm 0.797$ \\
\hline
\end{tabular}

Each value represents the mean \pm standard deviation in triplicate experiments. aAssay conditions: $100 \mathrm{mM}$ potassium carbonate buffer $(\mathrm{pH} 9.0)$ containing $30 \mathrm{mM}$ ammonium sulfate; $2 \mathrm{mM}, \mathrm{NAD}^{+} ; 9.7 \mu \mathrm{g} \mathrm{mL}{ }^{-1}$, AdhP; 1,3-propanediol concentration was varied from 1.17 to $600 \mathrm{mM}$; temperature was set at $37^{\circ} \mathrm{C}$.

bAssay conditions: $50 \mathrm{mM}$ Tris- $\mathrm{HCl}$ buffer $(\mathrm{pH} 7.0)$ containing $0.1 \mathrm{mM} \mathrm{NADH} ; 0.36 \mu \mathrm{gL}^{-1}$ AdhP; 3 HPA concentration was varied from 0.01 to $20 \mathrm{mM}$; temperature was set at $37^{\circ} \mathrm{C}$. CAssay conditions: $50 \mathrm{mM}$, potassium phosphate buffer ( $\mathrm{pH}$ 8.0) containing $1.0 \mathrm{mM}$ DTT; $2 \mathrm{mM}, \mathrm{NAD}^{+} ; 5.7 \mu \mathrm{g} \mathrm{mL}^{-1}$, AldD $\mathrm{Hb}$; 3HPA concentration was varied from 0.02 to $15 \mathrm{mM}$ temperature was set at $37^{\circ} \mathrm{C}$

(PHB/DCM) increased 108 and 455\%, respectively. The addition of $6 \mathrm{gL}^{-1}$ acetic acid resulted in the highest $3 \mathrm{HP}$, dry cell weight (DCM), and $\mathrm{PHB}$ content, reaching approximately $9.3 \mathrm{~g} \mathrm{~L}^{-1} 3 \mathrm{HP}$, $8 \mathrm{~g} \mathrm{~L}^{-1} \mathrm{DCM}$, and $40 \% \mathrm{PHB} / \mathrm{DCM}$ (Fig. 4c). The addition of $6 \mathrm{~g} \mathrm{~L}^{-1}$ acetic acid also resulted in a 1.5-fold increase in a total amount of $\mathrm{NAD}^{+}$and $\mathrm{NADH}$ and twofold increase in $\mathrm{NAD}^{+} / \mathrm{NADH}$ ratio compared with the result of $0 \mathrm{~g} \mathrm{~L}^{-1}$ acetic acid (Supplementary Fig. 11). Since $\mathrm{PhaB}$ of $H$. bluephagenesis is dependent on $\mathrm{NADH}$ for $\mathrm{PHB}$ synthesis, the synthesis of $\mathrm{PHB}$ consumes $\mathrm{NADH}^{55}$. The supply of acetic acid can increase acetylCoA pool. The excess NADH resulted from $3 \mathrm{HP}$ formation via 1,3propanediol oxidation can be balanced by accelerating $\mathrm{PHB}$ synthesis via a larger acetyl-CoA pool, this improves not only the
PHB synthesis but also glycolysis, TCA cycle, and 3HP synthesis (Supplementary Fig. 12). Therefore, L-aspartate as the precursor for the de novo biosynthesis of $\mathrm{NAD}^{+}$was possibly improved. As a result, both the total amount of $\mathrm{NAD}^{+}, \mathrm{NADH}$, and the $\mathrm{NAD}^{+}$/ $\mathrm{NADH}$ ratio were increased.

The $\mathrm{pH}$ in the cultures decreased from 9 to 5-6 after $24 \mathrm{~h}$ cultivation as the increase of $3 \mathrm{HP}$ in shake-flask inhibits cell growth and $3 \mathrm{HP}$ production. To stabilize $\mathrm{pH}$ above 7 after $24 \mathrm{~h}$ cultivation via improving the buffer capacity in the medium, the phosphate buffer was changed to $1.4 \% \mathrm{~K}_{2} \mathrm{HPO}_{4} \cdot 3 \mathrm{H}_{2} \mathrm{O}$ and $0.52 \%$ $\mathrm{KH}_{2} \mathrm{PO}_{4}$ in a modified minimal medium. This generated $12 \mathrm{~g} \mathrm{~L}^{-1}$ $3 \mathrm{HP}, 7 \mathrm{~g} \mathrm{~L}^{-1} \mathrm{DCM}$ and 50\% PHB/DCM from $H$. bluephagenesis TD27 grown in shake flasks, this was comparable to that of plasmid-expressing $H$. bluephagenesis TD $\Delta d d d A$ (p59) (Fig. 4d), along with an over $90 \%$ conversion ratio of 1,3-propanediol to 3HP (Supplementary Fig. 13).

The glucose level should be reduced to allow the minimum amount of glucose for efficient production of 3HP. Gradient concentrations of glucose $\left(5,10,15\right.$, and $\left.20 \mathrm{~g} \mathrm{~L}^{-1}\right)$ were co-fed with 20 or $30 \mathrm{~g} \mathrm{~L}^{-1} 1,3$-propanediol, respectively, to cultures of $H$. bluephagenesis TD27 (Fig. 4e). The addition of $5 \mathrm{~g} \mathrm{~L}^{-1}$ glucose and $20 \mathrm{~g} \mathrm{~L}^{-1} 1,3$-propanediol resulted in $\sim 18.9 \mathrm{~g} \mathrm{~L}^{-1} 3 \mathrm{HP}, 7.8 \mathrm{~g} \mathrm{~L}^{-1}$ DCM, and $47 \%$ PHB/DCM (Fig. 4e). In the presence of $5 \mathrm{~g} \mathrm{~L}^{-1}$ glucose and $20 \mathrm{~g} \mathrm{~L}^{-1}$ 1,3-propanediol, $3.5 \mathrm{~g} \mathrm{~L}^{-1}$ residual 1,3propanediol together with $0.2 \mathrm{~g} \mathrm{~L}^{-1}$ acetic acid were found in the culture together with less than $0.1 \mathrm{~g} \mathrm{~L}^{-1}$ residual glucose in the cultures of $H$. bluephagenesis TD27, indicating that the reduction of glucose enhanced 3HP production (Supplementary Fig. 14). An excess amount of $\mathrm{NADH}$ is formed during glycolysis and degradation of pyruvate to acetyl-CoA in the presence of sufficient 
a

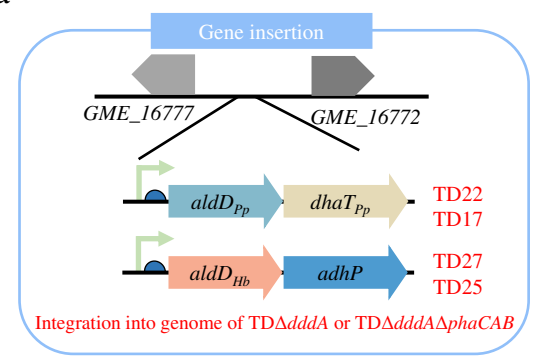

b

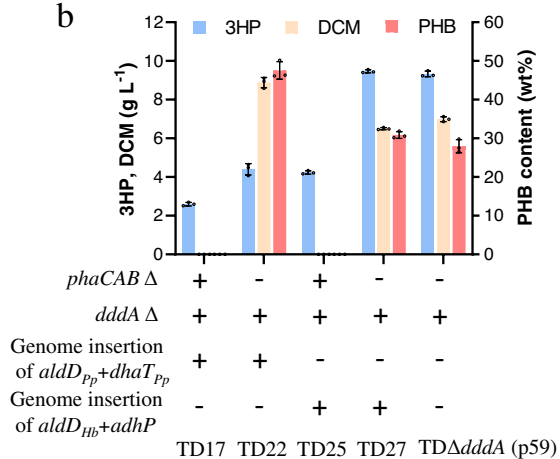

c

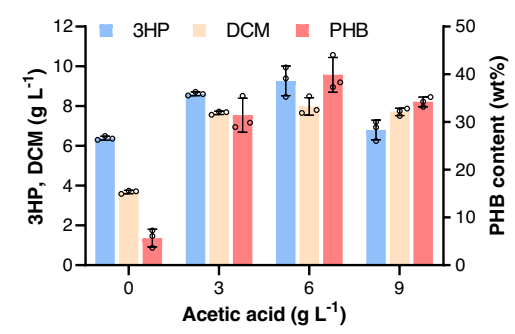

d

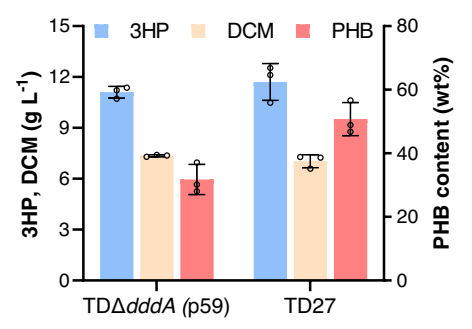

e

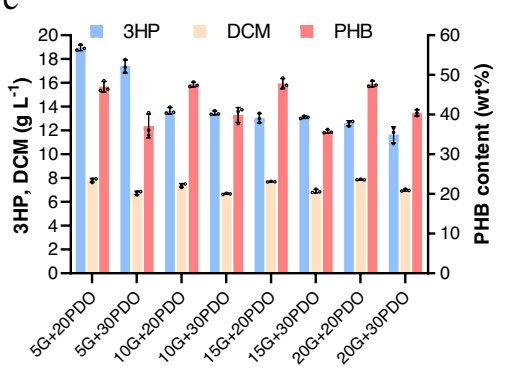

$\mathrm{f}$

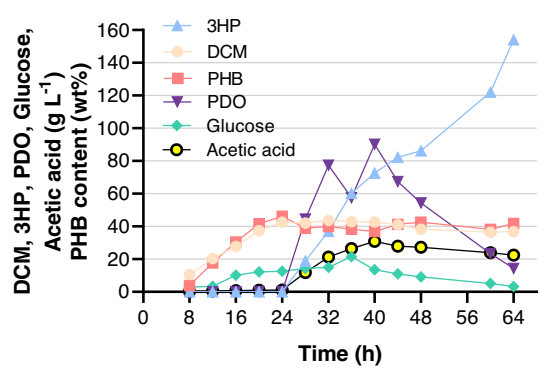

Fig. 4 Combinatorial optimization to increase 3HP production. a Genome engineering strategy and genetic constructs used for genomic integration.

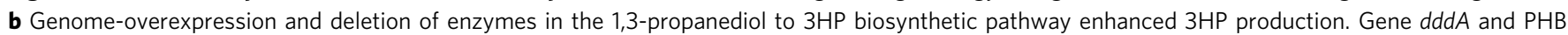

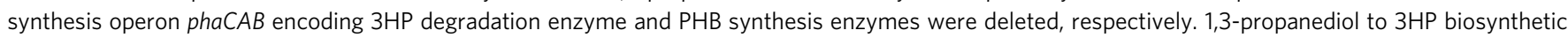
pathway of $P$. putida KT2440 and $H$. bluephagenesis were inserted into $H$. bluephagenesis TD $\Delta d d d A$ and $H$. bluephagenesis $\Delta d d d A \Delta$ phaCAB, respectively. Cells were grown in the defined minimal medium supplemented with $20 \mathrm{~g} \mathrm{~L}^{-1}$ glucose, $20 \mathrm{~g} \mathrm{~L}^{-1} 1,3$-propanediol, and $3 \mathrm{~g} \mathrm{~L}^{-1}$ acetic acid. c Characterization of $3 \mathrm{HP}$ and PHB produced by metabolically engineered $H$. bluephagenesis TD27 cultured at gradient concentrations of acetic acid to balance the redox state. Cells were grown in the defined minimal medium containing $20 \mathrm{~g} \mathrm{~L}^{-1}$ glucose, $20 \mathrm{~g} \mathrm{~L}^{-1}$ 1,3-propanediol, and gradient concentrations of acetic acid ( $\mathrm{g} \mathrm{L}^{-1}$ ) $0,3,6$, and 9 , respectively. The $\mathrm{pH}$ was adjusted to 9 after incubation for $24 \mathrm{~h}$. $\mathbf{d}$ Characterization of $3 \mathrm{HP}$ and $\mathrm{PHB}$ produced by metabolically engineered $\mathrm{H}$.

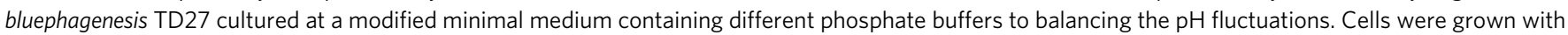

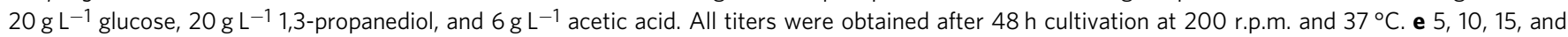
$20 \mathrm{~g} \mathrm{~L}^{-1}$ glucose (5G, 10G, 15G, and 20G) were co-fed with $20 \mathrm{~g} \mathrm{~L}^{-1} 1,3$-propanediol (20PDO) or 30 g L-1 1,3-propanediol (30PDO), respectively, to cultures of $H$. bluephagenesis TD27 grown in a modified minimal medium containing $6 \mathrm{~g} \mathrm{~L}^{-1}$ acetic acid. $\mathbf{f}$ Time profiles of cell growth (DCM), PHB content, and concentrations of carbon sources (glucose, 1,3-propanediol, acetic acid), and 3HP production during the fed-batch culture of $H$. bluephagenesis TD27. $60 \mathrm{~g} \mathrm{~L}^{-1}$ 1,3-propanediol and $6 \mathrm{~g} \mathrm{~L}^{-1}$ acetic acid were added at 24,32 and $38 \mathrm{~h}$ during fed-batch culture, respectively. For the shake-flask cultivation, The initial $\mathrm{pH}$ of all shake-flask studies was 9. All data represent the mean of $n=3$ biologically independent samples and error bars show s.d.

glucose. The excessive NADH condition associated with $3 \mathrm{HP}$ formation and glycolysis can in turn inhibit glycolysis and $3 \mathrm{HP}$ synthesis (Supplementary Fig. 12). Thus, a reduced amount of glucose may reduce the $\mathrm{NADH}$ produced by glycolysis during $3 \mathrm{HP}$ synthesis, resulting in more conversion of 1,3-propanediol to $3 \mathrm{HP}$.

E. coli and $H$. bluephagenesis were transformed using plasmids p30 and p59 expressing $\mathrm{AldD}_{\mathrm{Pp}}$ and $\mathrm{DhaT}_{\mathrm{Pp}}$ cloned from P. putida KT2440, $\mathrm{AldD}_{\mathrm{Hb}}$ and AdhP from $H$. bluephagenesis, respectively. E. coli was cultured in a modified minimal medium containing $1 \% \mathrm{NaCl}$, and $H$. bluephagenesis in another modified minimal medium containing $6 \% \mathrm{NaCl}$. Recombinant E. coli harboring alcohol dehydrogenase (AdhP) and aldehyde dehydrogenase $\left(\mathrm{AldD}_{\mathrm{Hb}}\right)$ cloned from $H$. bluephagenesis produced only $0.7 \mathrm{~g} \mathrm{~L}^{-1} 3 \mathrm{HP}$. In contrast, the $H$. bluephagenesis TD $\Delta d d d A$ (p59) generated $11 \mathrm{~g} \mathrm{~L}^{-1} 3 \mathrm{HP}$ compared to only $5 \mathrm{~g} \mathrm{~L}^{-1} 3 \mathrm{HP}$ secreted by $H$. bluephagenesis TD $\Delta d d d A$ (p30) harboring the DhaT $_{\mathrm{Pp}}$ and $\mathrm{AldD}_{\mathrm{Pp}}$ from P. putida KT2440 (Supplementary Fig. 15).

To further enhance $3 \mathrm{HP}$ production, the fed-batch fermentation process was optimized employing various feeding strategies, including continuous or intermittent feeding strategy during the late period of growth. During the continuous feeding process, cell growth was inhibited when 1,3-propanediol and glucose were fed at $8 \mathrm{~h} .60 \mathrm{~g} \mathrm{~L}^{-1} 3 \mathrm{HP}$ with $<30 \mathrm{~g} \mathrm{~L}^{-1} \mathrm{DCM}$ were obtained from cultures of H. bluephagenesis TD27 (Supplementary Fig. 16). The production of $3 \mathrm{HP}$ will convert a large amount of $\mathrm{NAD}^{+}$into $\mathrm{NADH}$ during the logarithmic growth phase, which changes the redox balance. Excessive NADH generates feedback inhibition to glycolysis, negatively affecting cellular catabolism and growth. In addition, the enzyme activity of $\mathrm{AldD}_{\mathrm{Hb}}$ was observed to decrease over time, and the activity of AdhP was observed to increase up to $24 \mathrm{~h}$ (Supplementary Fig. 17). When $60 \mathrm{~g} \mathrm{~L}^{-1}$ 1,3-propanediol was added at 24,28 , and $32 \mathrm{~h}$ during fed-batch fermentation process to separate cell growth and 3HP production, the engineered $H$. bluephagenesis TD27 produced $154 \mathrm{~g} \mathrm{~L}^{-1} 3 \mathrm{HP}$ with a yield and productivity of $0.93 \mathrm{~g} \mathrm{~g}^{-1} 1,3$-propanediol and $2.4 \mathrm{~g} \mathrm{~L}^{-1} \mathrm{~h}^{-1}$, respectively (Fig. $4 \mathrm{f}$ ).

Engineering $H$. bluephagenesis TD27 for productions of 3HP and its copolymers. Polyhydroxybutyrate or poly-3-hydroxybutyrate (PHB), is very brittle due to its high crystallinity that limits its application. In contrast, poly-3-hydroxypropionate (P3HP) shows good combined mechanical properties including an elongation at break of more than $600 \%$ and Young's modulus of $300 \mathrm{MPa}-3$ $\mathrm{GPa}^{56}$. The low melting temperature $T_{\mathrm{m}}$ of $\mathrm{P} 3 \mathrm{HP}$ is $<100^{\circ} \mathrm{C}$, which limits its stability in a higher temperature environment. On the other hand, the $T_{\mathrm{m}}$ of $\mathrm{PHB}$ is high which can reach $176^{\circ} \mathrm{C}$. Thus, copolymerization of $3 \mathrm{HB}$ and $3 \mathrm{HP}$ compensate for the weak properties 

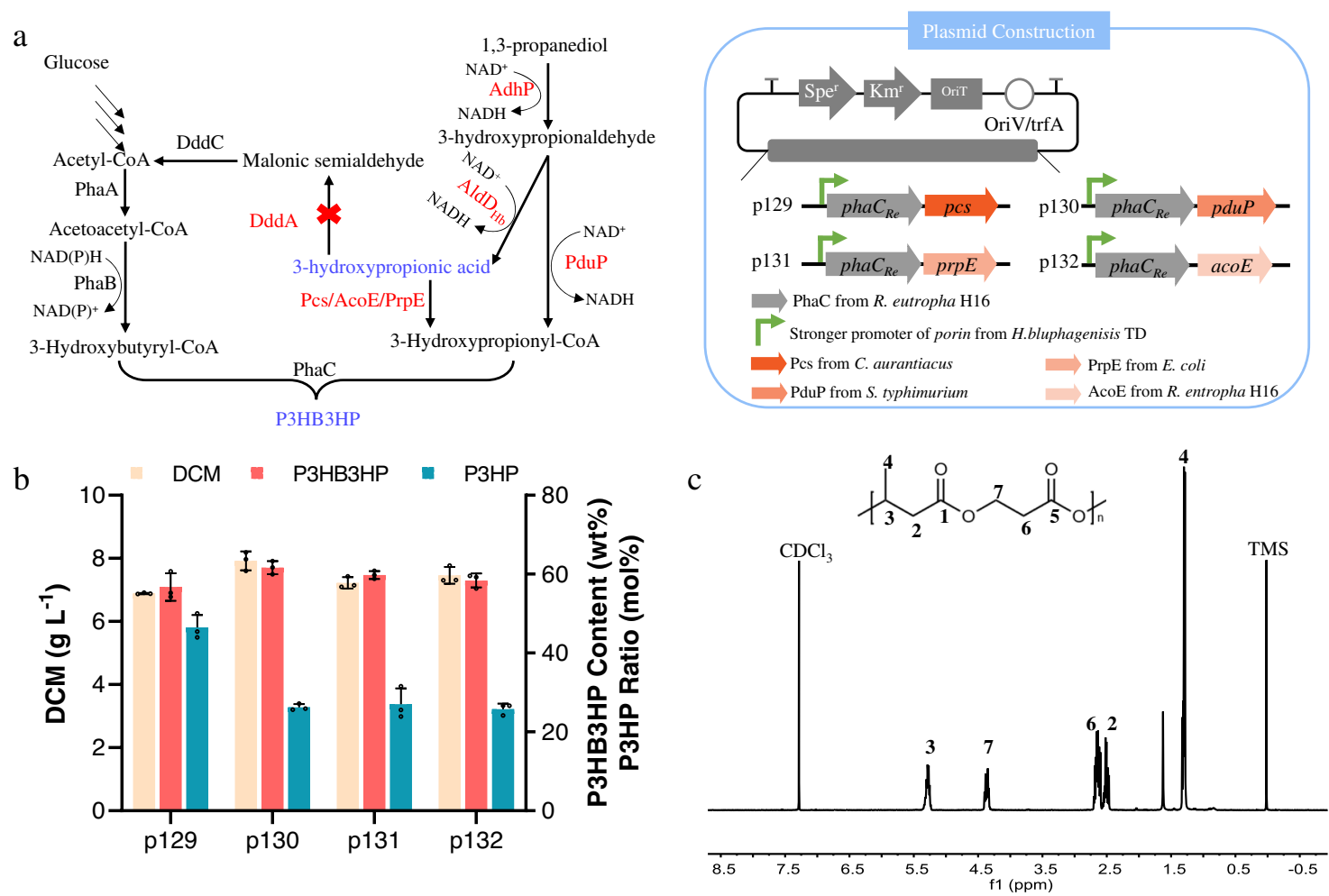

Fig. 5 Enzyme screening for improving biosynthesis of P3HB3HP by engineered $\boldsymbol{H}$. bluephagenesis TD27. a Strategies for microbial co-production of 3HP and $\mathrm{P} 3 \mathrm{HB} 3 \mathrm{HP}$ involving a combination of genome-based gene expression and plasmid-based gene expression. Red color " $\mathrm{X}$ " indicates the inactivation of metabolic pathways. Gene adhP encodes 1,3-propanediol dehydrogenase from $H$. bluphagenesis, ald $D_{H b}$ encodes aldehyde dehydrogenase from $H$. bluphagenesis, pcs encodes coenzyme A ligase domain from Chloroflexus aurantiacus, pduP encodes propionaldehyde dehydrogenase from Salmonella typhimurium, prpE encodes propionyl-CoA synthase from E. coli, acoE encodes acetyl-CoA synthase from Ralstonia entropha. Genes were expressed under the control of constitutive promoters in a multiple-copy episomal plasmid pKS. b P3HB3HP contents and monomer compositions generated by engineered H. bluephagenesis TD27 overexpressing different 3-hydroxypropionyl-CoA biosynthetic genes. Cells were grown in the modified minimal medium containing $20 \mathrm{~g} \mathrm{~L}^{-1}$ glucose, $10 \mathrm{~g} \mathrm{~L}^{-1}$ 1,3-propanediol, and $6 \mathrm{~g} \mathrm{~L}^{-1}$ acetic acid. All titers were obtained after $48 \mathrm{~h}$ cultivation at $200 \mathrm{r}$.p.m. and $37^{\circ} \mathrm{C}$. The initial $\mathrm{pH}$ of all shake-flask studies was 9 . All data represent the mean of $n=3$ biologically independent samples and error bars show s.d. $\mathbf{c}{ }^{1} \mathrm{H}$ NMR of $\mathrm{P}$ (3HB-co-40\% 3HP) copolymers produced by the engineered H. bluephagenesis TD27 overexpressing pcs. TMS tetramethylsilane (internal standard).

of each homopolymers ${ }^{57}$. Since recombinant $H$. bluephagenesis produced $3 \mathrm{HP}$ efficiently, 3HP-related copolymers are expected to be produced appropriately. It was reasonable to assume that adequate $3 \mathrm{HP}-\mathrm{CoA}$ and appropriate $\mathrm{PhaC}$ polymerase are essential for the accumulation of copolymer P3HB3HP consisting of 3hydroxybutyrate $(3 \mathrm{HB})$ and $3 \mathrm{HP}$, given the $\mathrm{PHB}$ is natively synthesized by $H$. bluephagenesis (Fig. 5a). To produce P3HB3HP, coenzyme A ligase domain (Pcs) from Chloroflexus aurantiacus, propionyl-CoA synthase (PrpE) from E. coli, acetyl-CoA synthase (AcoE) from Ralstonia entropha, and propionaldehyde dehydrogenase (PduP) from Salmonella typhimurium, were selected to generate 3HP-CoA from 3-hydroxypropionic acid and from 3hydroxypropionaldehyde, respectively. PhaC polymerase from $R$. entropha was chosen for its strong $3 \mathrm{HP}$ polymerization ability when expressed in other bacteria including E. coli (Fig. 5a) ${ }^{58}$.

The strong $3 \mathrm{HP}$ producer $H$. bluephagenesis TD27 was then transformed with the four constructs, and $\mathrm{P} 3 \mathrm{HB} 3 \mathrm{HP}$ was successfully produced in $7 \mathrm{~g} \mathrm{~L}^{-1}$ DCM containing approximately $60 \%$ copolymer (Fig. 5b). H. bluephagenesis TD27 overexpressing $\mathrm{Pcs}$ and $\mathrm{PhaC}$ reached $45 \% 3 \mathrm{HP}$ molar ratio in the $\mathrm{P} 3 \mathrm{HB} 3 \mathrm{HP}$ content. The extracellular $3 \mathrm{HP}$ yield increased gradually with the increase of 1,3-propanediol concentration in the cell cultures. The $\mathrm{P} 3 \mathrm{HB} 3 \mathrm{HP}$ content and $\mathrm{P} 3 \mathrm{HP}$ monomer composition increased to a maximum of 54 and $45 \%$, respectively, when $12 \mathrm{gL}^{-1} 1,3$ propanediol was added to the cultures (Supplementary Fig. 18). The glucose and 1,3-propanediol concentrations were optimized to find the minimum amount of glucose and 1,3-propanediol for efficient production of $\mathrm{P} 3 \mathrm{HB} 3 \mathrm{HP}$. The $\mathrm{P} 3 \mathrm{HP}$ molar ratios in P3HB3HP were found to be between 32 and $40 \%$ when different concentrations of 1,3-propanediol $\left(2,4\right.$, and $\left.8 \mathrm{~g} \mathrm{~L}^{-1}\right)$ were added, respectively. The DCM and $\mathrm{P} 3 \mathrm{HB} 3 \mathrm{HP}$ content increased with the increase of glucose concentration (Supplementary Fig. 19). The fed-batch production of $\mathrm{P} 3 \mathrm{HB} 3 \mathrm{HP}$ allowed a $60 \%$ polymer accumulation with the $\mathrm{P} 3 \mathrm{HP}$ ratio reaching $40 \%$ in the copolymers (Supplementary Fig. 20).

The composition of a $\mathrm{P} 3 \mathrm{HB} 3 \mathrm{HP}$ was studied using NMR (Fig. 5c). Two characteristic peaks of P3HP at $\delta=2.59-2.70$ and $\delta=4.31-4.39 \mathrm{ppm}$ (peaks 6 and 7) were identified, together with three peaks of $\mathrm{P} 3 \mathrm{HB}$ at $\delta=1.24-1.33, \delta=2.47-2.56$ and $\delta=$ 5.23-5.35 ppm (peaks 4, 2, and 3). The molar ratio of P3HP in this copolymer has been shown can achieve approximately $40 \%$ when calculated by integrating the areas of specific related signals.

\section{Discussion}

$H$. bluephagenesis and its recombinants have been demonstrated to be economic chassis for reducing cost for bulk chemicals ${ }^{37}$. A systematic metabolic engineering approach was taken to achieve efficient production by deleting the $3 \mathrm{HP}$ degradation pathway combined with overexpression of $\mathrm{DhaT}_{\mathrm{Pp}}$ and $\mathrm{AldD}_{\mathrm{Pp}}$ proteins from $P$. putida KT2440 for $3 \mathrm{HP}$ production in $H$. bluephagenesis grown in the presence of 1,3-propanediol (Fig. 1a). Moreover, based on the RNA-seq data, the $a d h P$ and $a l d D_{H b}$ genes were chosen and overexpressed for enhanced catalytic activities toward 
3HP flux (Fig. 2). The two combinations of genes along with promoter of phaCAB operon from Ralstonia eutropha H16 and stronger RBS were inserted into the genome of the Halomonas chassis to approach better production performance (Fig. 4a, b). Combining with balancing the intracellular redox state via acetic acid uptake, the recombinant chassis can synthesize $154 \mathrm{~g} \mathrm{~L}^{-1}$ $3 \mathrm{HP}$ during the fed-batch growth (Fig. 4f).

Since 3HP based polyhydroxyalkanoates (PHA) polyesters provide improved mechanical strengths for $\mathrm{PHA}^{59}$, it has become an attractive idea to make $3 \mathrm{HP}$ based PHA. In this study, P3HP3HB copolymer was produced based on the established $3 \mathrm{HP}$ synthesis pathway combined with overexpression of $p c s$ and phaC, two heterologous genes (Fig. 5a). The resulting $\mathrm{P} 3 \mathrm{HP} 3 \mathrm{HB}$ copolymer contained a 45\% P3HP molar ratio when fed with 1,3propanediol. The P3HP ratio also can be tuned from 32 to $40 \%$ by varying the co-feeding concentrations of 1,3-propanediol and glucose. For the first time, engineered Halomonas sp. has been employed to produce $\mathrm{P} 3 \mathrm{HB} 3 \mathrm{HP}$.

The selected substrate 1,3-propanediol contributed to the high 3HP titer (Fig.1a). As mentioned above, 3HP production via metabolic engineering started from 1,3-propanediol could avoid the paradox between glycerol dehydratase and aldehyde dehydrogenase activities, which generate from the imbalance of vitamin $\mathrm{B}_{12}$ synthesis and $\mathrm{NAD}^{+}$regeneration. Under the aerobic catalysis of 1,3-propanediol oxidoreductase and aldehyde dehydrogenase, 3HP was formed via the two-step bio-catalysis (Fig. 1a) ${ }^{23}$. This study proposed to use 1,3-propanediol as a substrate for 3HP production. As 1,3-propanediol can be produced efficiently from glycerol, a biodiesel production byproduct under an anaerobic bioprocess, it is cost-effective ${ }^{60,61}$. Under the 1,3-propanediol production conditions, 3HP was outcompeted with relatively low titer due to the low aldehyde dehydrogenase enzyme activity under anaerobic conditions ${ }^{6}$. In this study, 1, 3propanediol was converted into $3 \mathrm{HP}$, thus bridging low-cost glycerol to high-valued 3HP efficiently. Also, fewer secondary byproducts were generated in the traditional processes under aerobic fed-batch condition, especially lactic acid, which is difficult to be separated from $3 \mathrm{HP}$ in the culture ${ }^{6}$.

In this study, glucose was added to support cell growth and PHB synthesis. The synthesis of $3 \mathrm{HP}$ is derived from 1,3-propanediol (Supplementary Fig. 12). Since crude 1,3-propanediol (not the purified one for making polymer PTT) produced from the microbial conversion of crude glycerol (from biodiesel production) is sold on Chinese local market with a price of $\$ 600 /$ ton, conversion of 1,3-propanediol to $3 \mathrm{HP}$ is almost $100 \%$, the material cost of $3 \mathrm{HP}$ should be around $\$ 600 /$ ton excluding other minimal substrate costs. Since $3 \mathrm{HP}$ has not been available in large scale on the market, its price is offered based on grams. This is not much value as references for bulk chemicals. However, P3HP3HB with an improved material property over the existing PHBV sold at a price of $\$ 7000 /$ ton, should have a much high market value even though 1,3-propanediol is used as a co-substrate.

When PHB production came from consumption of glucose, $3 \mathrm{HP}$ yield was increased from 4.2 to $6.4 \mathrm{~g} \mathrm{~L}^{-1}$, and $\sim 0.21 \mathrm{~g} \mathrm{~L}^{-1}$ PHB was produced from $5.8 \mathrm{~g} \mathrm{~L}^{-1}$ glucose (Supplementary Table 4). Theoretically, the conversion of $1 \mathrm{M}$ glucose to acetylCoA can produce $4 \mathrm{M} \mathrm{NADH}$, and $1 \mathrm{M} 3 \mathrm{HB}-\mathrm{CoA}$ is generated from $2 \mathrm{M}$ acetyl-CoA, diverting acetyl-CoA away from the TCA cycle where much of the $\mathrm{NADH}$ is generated, reducing the formation of $3 \mathrm{M} \mathrm{NADH}$. Therefore, $0.21 \mathrm{~g} \mathrm{~L}^{-1} \mathrm{PHB}$ (approximately $2.44 \mathrm{mM} 3 \mathrm{HB}-\mathrm{CoA}$ ) can reduce $7.32 \mathrm{mM} \mathrm{NADH}$ formation. The $3 \mathrm{HP}$ yield was improved by the regeneration of $\mathrm{NAD}^{+}$(via consuming NADH). The excess NADH resulted from $3 \mathrm{HP}$ formation via the 1,3-propanediol oxidation feedback inhibits the glycolysis and $3 \mathrm{HP}$ production. On the other hand, $\mathrm{PHB}$ production from glucose diverts acetyl-CoA away from the TCA cycle. The decrease in anabolism resulting from a weakened TCA leads to the accumulation of NADPH. The increased NADPH inhibits glucose 6-phosphate dehydrogenase activity, resulting in the accumulation of glucose 6-phosphate, thus causing a lower glucose uptake rate. Therefore, the total amount of glucose used reduced $3 \mathrm{~g} \mathrm{~L}^{-1}(16.67 \mathrm{mM})$ compared with no $\mathrm{PHB}$ production (Supplementary Table 4), further reducing the formation of 66.68 mM NADH. In conclusion, $74 \mathrm{mM}$ (66.68 plus 7.32$) \mathrm{NAD}^{+}$is saved for $3 \mathrm{HP}$ production. Compared with no $\mathrm{PHB}$ production, the yield of $3 \mathrm{HP}$ should be increased by $3.3 \mathrm{~g} \mathrm{~L}^{-1}$. In fact, $3 \mathrm{HP}$ yield increased by $2.2 \mathrm{~g} \mathrm{~L}^{-1}$. This may be due to the excess $\mathrm{NADH}$ via the 1,3-propanediol oxidation that also feedback inhibits the $3 \mathrm{HP}$ production.

When $\mathrm{PHB}$ production from both glucose and acetic acid, 3HP yield was increased from 6.4 to $9.3 \mathrm{~g} \mathrm{~L}^{-1}$, and $\sim 3.2 \mathrm{~g} \mathrm{~L}^{-1} \mathrm{PHB}$ was produced from $10.5 \mathrm{~g} \mathrm{~L}^{-1}$ glucose and $4 \mathrm{~g} \mathrm{~L}^{-1}$ acetic acid (Supplementary Table 4). Theoretically, $1 \mathrm{M} \mathrm{NADH}$ is needed to produce $1 \mathrm{M} 3 \mathrm{HB}$-coA from $2 \mathrm{M}$ acetic acid regardless of the ATP consumption during the conversion of acetate to acetyl-CoA. Therefore, $4 \mathrm{~g} \mathrm{~L}^{-1}$ acetic acid $(66.67 \mathrm{mM})$ can consume 33.33 $\mathrm{mM}$ NADH and produce $33.33 \mathrm{mM} 3 \mathrm{HB}-\mathrm{CoA}\left(\sim 2.9 \mathrm{~g} \mathrm{~L}^{-1} \mathrm{PHB}\right)$. Compared with $\mathrm{PHB}$ production from glucose, the yield of $3 \mathrm{HP}$ should be increased by $1.5 \mathrm{~g} \mathrm{~L}^{-1}$. In fact, 3HP yield increased by $2.9 \mathrm{~g} \mathrm{~L}^{-1}$. This may be attributed to the utilization of ATP that can also promote the consumption of NADH by the respiratory chain. When acetate is added, the consumption of $\mathrm{NADH}$ improves 3HP synthesis and glycolysis by accelerating $\mathrm{PHB}$ synthesis from a bigger acetyl-CoA pool, the true cell mass increased from 3.5 to $4.8 \mathrm{~g} \mathrm{~L}^{-1}$. As a result, $3 \mathrm{HP}$ be produced at $9.3 \mathrm{~g} \mathrm{~L}^{-1}$ with the yield of $1.8 \mathrm{~mol} \mathrm{~mol}^{-1}$ glucose from flask scale studies (Supplementary Table 4). When the amount of glucose used was $5 \mathrm{~g} \mathrm{~L}^{-1}, 3 \mathrm{HP}$ was produced at $18.9 \mathrm{~g} \mathrm{~L}^{-1}$ with the yield of $7.6 \mathrm{~mol} \mathrm{~mol}^{-1}$ glucose on flask scales (Supplementary Fig. 14).

Since $H$. bluephagenesis is able to grow under an unsterilized conditions with a high tolerance to a high concentration of salt, in this case, it is salt of $3 \mathrm{HP}$, the chassis is promising for industrial production of high titer extracellular small molecules including threonine, succinate, and itaconate ${ }^{62,63}$. In contrast, the most commonly used bacterium, E. coli, demonstrated to have a growth inhibition at $10 \mathrm{~g} \mathrm{~L}^{-1} 3 \mathrm{HP}$ in its culture ${ }^{64}$. H. bluephagenesis has been used to produce several PHA including PHB, PHBV, and P3HB4HB. It could be further extended for more PHA synthesis since many PDORs and ALDHs possess broad substrate specificities with the potentials for synthesizing other monomers ${ }^{65,66}$. Poly(3-hydroxybutyrate-co-5-hydroxyvderate) (P3HB5HV) and Poly(3-hydroxybutyrate-co-6-hydroxyhexanoate) (P3HB6HHX) could also be produced by supplementing corresponding diols to cultures of $H$. bluephagenesis based on the polymer biosynthetic methods mentioned above as some broad specificity CoA transferase and $\mathrm{PhaC}$ have been reported ${ }^{67}$. Moreover, it can be assumed that pure $\mathrm{P} 3 \mathrm{HP}$ can be produced by deleting $\mathrm{PHB}$ synthesis pathway. Since $\mathrm{PhaB}$ of $H$. bluephagenesis is dependent on $\mathrm{NADH}$ as a cofactor for PHB synthesis, the PHB accumulation can further consume NADH, thus facilitating further synthesis of 3HP. The 3HP monomer synthesis decreased once the deletion of PHB synthesis pathway is completed. In order to produce pure P3HP, the introduction of other metabolic pathways to regenerate $\mathrm{NAD}^{+}$can promote $3 \mathrm{HP}$ synthesis after the loss of $\mathrm{PHB}$ synthesis pathways. Further investigations could generate more unusual PHA from this chassis.

In conclusion, this study established an effective $3 \mathrm{HP}$ production strategy using 1,3-propanediol as a substrate by halophilic chassis $H$. bluephagenesis, demonstrating this strain to be an excellent chassis. The $3 \mathrm{HP}$ has also been found to be able to become a monomer of the PHA copolymer P3HP3HB. H. bluephagenesis, as a platform for the next generation industrial 
biotechnology, holds a bright prospect for low-cost productions of both extracellular and intracellular products.

\section{Methods}

Bacterial strains and growth conditions. Strains and plasmids used in this study are listed in Supplementary Tables 1 and 2. Primers used in this study are listed in Supplementary Data 1. Halomonas bluephagenesis strain TD01, the wild-type isolated from Aydingol Lake of Xinjiang province in China, has been deposited in the China General Microbiological Culture Collection Center (CGMCC) under the collection number 4353. Escherichia coli S17-1 was used as the host for plasmid construction and the conjugation donor. The plasmids were constructed using Gibson Assembly. Plasmid extraction kits were purchased from Tiangen Biotech Co., Ltd. (Beijing, China). Plasmids for expression were electroporated into E. coli S17-1. DNA fragments were amplified using Q5 ${ }^{\circledR}$ High-Fidelity DNA polymerase (New England Biolabs Inc., USA).

For molecular biological studies, E. coli was cultured at $37^{\circ} \mathrm{C}$ in an LB medium containing $\left(\mathrm{g} \mathrm{L}^{-1}\right) 10 \mathrm{NaCl}$ (Analytical reagent, Sinopharm Chemical Reagent Co., Ltd., China), 10 tryptone (Analytical reagent, Oxoid, England), and 5 yeast extract (Analytical reagent, Oxoid, England). H. bluephagenesis was cultured at $37^{\circ} \mathrm{C}$ in a 60-LB medium, namely, the LB medium supplemented with $60 \mathrm{~g} \mathrm{~L}^{-1} \mathrm{NaCl}$. A 20LB medium indicates the LB medium containing $20 \mathrm{~g} \mathrm{~L}^{-1} \mathrm{NaCl}$. Moreover, $15 \mathrm{~g} \mathrm{~L}^{-1}$ agar (BioDee Biotechnology Co., Ltd., Beijing, China) was added before autoclaving for preparing solid media in Petric plates. Ampicillin $(100 \mu \mathrm{g} \mathrm{mL}-1)$,

chloramphenicol $\left(25 \mu \mathrm{g} \mathrm{mL}^{-1}\right)$, kanamycin $\left(50 \mu \mathrm{g} \mathrm{mL}^{-1}\right)$, or spectinomycin $\left(100 \mu \mathrm{g} \mathrm{mL}^{-1}\right)$, all purchased from BioDee Biotechnology Co., Ltd. (Beijing, China), were added to the above media whenever necessary. Absolute ethanol (Analytical reagent, Beijing Tongguang Chemicals Co., China) and chloroform (Analytical reagent, Beijing Tongguang Chemicals Co., China) were used for PHA extraction and analysis. $3 \mathrm{HPA}, \mathrm{NADH}$, and $\mathrm{NAD}^{+}$were purchased from Shanghai Kaiwei Biochemical Co., Ltd. and Abmole (Shanghai, China). Unless indicated otherwise, 1,3-propanediol and all other chemicals were obtained from Shanghai Sangon Biotech Co., Ltd.

Genome editing using CRISPR/Cas9. Gene insertions and deletions on the chromosome of $H$. bluephagenesis were conducted using CRISPR/Cas9 based on homologous recombination ${ }^{33,68}$. After PCR verification and DNA sequencing, the engineered $H$. bluephagenesis TD with $d d d A$ gene deleted was designated as H. bluephagenesis TD $\triangle d d d A$. H. bluephagenesis TD01 deleted with its native PHA synthesis genes phaA, phaB and phaC is named as H. bluephagenesis TD $\triangle$ phaCAB. $H$. bluephagenesis TD $\triangle p h a C A B$ deleted with $d d d A$ gene is further named as H. bluephagenesis TD $\Delta$ phaCABAdddA. H. bluephagenesis TD $\Delta$ phaCABAdddA and H. bluephagenesis TD $\Delta d d d A$ inserted with $d h a T_{P p}$ and ald $D_{P p}$ gene from Pseudomonas putida KT2440 under the control of $\mathrm{P}_{\mathrm{Re}}$ promoter in $\mathrm{G} 4$ site on its genome are renamed as $H$. bluephagenesis TD17 and H. bluephagenesis TD22, respectively. $H$. bluephagenesis TD $\triangle p h a C A B \triangle d d d A$ and $H$. bluephagenesis TD $\Delta d d d A$ inserted with adhP and ald $D_{H b}$ gene from $H$. bluephagenesis under the control of $\mathrm{P}_{\mathrm{Re}}$ promoter on the G4 site are renamed as H. bluephagenesis TD25 and H. bluephagenesis TD27, respectively.

Conjugation. Conjugation was performed as an efficient way to transform plasmids into H. bluephagenesis ${ }^{37}$. E. coli S17-1, with plasmids electro-transformed in, were used as donors. The donors and $H$. bluephagenesis recipient cells were cultured at $37^{\circ} \mathrm{C}$ for $4 \mathrm{~h}$ till the optical density $\left(\mathrm{OD}_{600}\right)$ reached $0.3-0.5$. After collecting $1 \mathrm{~mL}$ of cells of E. coli S17-1 and H. bluephagenesis by centrifugation respectively, the pellet cells were washed twice using a 20-LB medium. Then the cells were re-suspended and mixed at a ratio 1:1, followed by incubation on 20-LB plates at $37^{\circ} \mathrm{C}$ for $12 \mathrm{~h}$. The mixed bacteria were spread on plates containing corresponding antibiotics and incubated at $37^{\circ} \mathrm{C}$ for $24-48 \mathrm{~h}$ for single colonies, which were verified by PCR for future use. H. bluephagenesis wild-type and recombinant strains were stored in autoclaved $25 \%(\mathrm{v} / \mathrm{v})$ glycerol at $-80^{\circ} \mathrm{C}$ if needed.

Shake-flask studies. For shake-flask PHA production, a defined minimal medium containing $6 \% \mathrm{NaCl}, 0.05 \%$ urea, $0.02 \% \mathrm{MgSO}_{4}, 1.0 \% \mathrm{Na}_{2} \mathrm{HPO}_{4} \cdot 12 \mathrm{H}_{2} \mathrm{O}, 0.15 \%$ $\mathrm{KH}_{2} \mathrm{PO}_{4}, 1.0 \%$ trace element solution I, $0.1 \%$ trace element solution II and $0.1 \%$ yeast extract, was used. Phosphate buffer was changed to $1.4 \% \mathrm{~K}_{2} \mathrm{HPO}_{4} \cdot 3 \mathrm{H}_{2} \mathrm{O}$ and $0.52 \% \mathrm{KH}_{2} \mathrm{PO}_{4}$ in a modified minimal medium. The $\mathrm{pH}$ of the solution was adjusted to 8.5-9.0 using $5 \mathrm{M} \mathrm{NaOH}$. Trace element solution I comprises $0.5 \% \mathrm{Fe}$ (III)- $\mathrm{NH}_{4}$-citrate and $0.2 \% \mathrm{CaCl}_{2}$ dissolved in $1 \mathrm{M} \mathrm{HCl}$; Trace element solution II contains $0.01 \% \mathrm{ZnSO}_{4} \cdot 7 \mathrm{H}_{2} \mathrm{O}, 0.003 \% \mathrm{MnCl}_{2} \cdot 4 \mathrm{H}_{2} \mathrm{O}, 0.03 \% \mathrm{H}_{3} \mathrm{BO}_{3}, 0.02 \% \mathrm{mg} \mathrm{L}^{-1}$ $\mathrm{CoCl}_{2} \cdot 6 \mathrm{H}_{2} \mathrm{O}, 0.003 \% \mathrm{NaMoO}_{4} \cdot 2 \mathrm{H}_{2} \mathrm{O}, 0.002 \% \mathrm{NiCl}_{2} \cdot 6 \mathrm{H}_{2} \mathrm{O}$, and $0.001 \%$

$\mathrm{CuSO}_{4} \cdot 5 \mathrm{H}_{2} \mathrm{O}$ dissolved in $1 \mathrm{M} \mathrm{HCl}$. Glucose was added at a final concentration of $3 \%$ at the beginning of the experiment. While 1,3-propanediol (Analytical reagent, Tianjin Guangfu Chemicals Co., China) was added at $0.5 \%$ or other concentrations as indicated.

For all shake-flask studies, the microbial glycerol stocks were resuscitated by streaking on fresh $60-\mathrm{LB}$ plates. Single colonies from streaked or newly-conjugated plates were picked and inoculated in the $60-\mathrm{LB}$ medium for $24 \mathrm{~h}$ at $200 \mathrm{rpm}$ to acquire the first seed culture, which was further grown on a fresh 60-LB liquid medium at a volume ratio of $1 \%$. The second seed culture was inoculated for $10 \mathrm{~h}$ at $200 \mathrm{rpm}$ until its $\mathrm{OD}_{600}$ reached 1.0. Afterward, it was inoculated into $500-\mathrm{mL}$ conical flasks containing $50 \mathrm{~mL}$ of the defined minimal medium at a volume ratio of $5 \%$ and cultivated for $48 \mathrm{~h}$ at $200 \mathrm{rpm}$. Antibiotics were added if needed. The temperature for all cultivations was $37^{\circ} \mathrm{C}$. All data were analyzed and graphed by GraphPad Prism 8.

RNA sequencing and quantitative RT-PCR. The bacteria were sampled from shake-flask experiments after $12 \mathrm{~h}$ cultivation. Then, the samples were centrifuged at $4000 \times g$ and $4{ }^{\circ} \mathrm{C}$ for $10 \mathrm{~min}$ followed by quick-frozen in liquid nitrogen. For RNA sequencing and analysis, they were transported immediately to the testing company in dry ice (solid carbon dioxide). RNA extraction, sequencing, and analysis were performed by Hangzhou Lianchuan Bio Technologies Co. Ltd. Briefly, RNA was extracted by TRIzol ${ }^{\oplus}$ Reagent (Invitrogen, U.S.A). The RNA library was constructed using Illumina Truseq ${ }^{\mathrm{T} M}$ RNA sample prep Kit (Illumina, U.S.A). Sequencing was performed using the Illumina Hiseq platform after the processing of libraries via Truseq SBS Kit v3-HS (Illumina, U.S.A). The expression differences were analyzed using edgeR.

For quantitative RT-PCR, total RNA was isolated from bacterial culture using TRIzol $^{\oplus}$ Reagent according to the manufacturer's instructions. RNA concentration was determined via spectrophotometry at $260 \mathrm{~nm}$. Removal of genomic DNA and synthesis of cDNA were carried out using PrimeScript RT reagent Kit with gDNA Eraser (Takara). qRT-PCR was conducted using $2 \times$ RealStar Green Power Mixture with ROX II (Genstar, China) with the 7500 Fast Real-Time PCR System (Applied Biosystems). Constitutively transcribed 16s rRNA was used as a reference control to normalize the total RNA quantity of different samples. The relative difference of mRNA level was calculated using the $\Delta \Delta \mathrm{Ct}$ method ${ }^{69}$. Two independent biological samples with three technical repeats for each sample were performed for each qRTPCR analysis.

Protein expression and enzyme activities. Plasmids p90, p91, p92, and p98 were constructed encoding genes $a d h P$, ald $D_{H b}$, dha $T_{P p}$ with a His-tag on C-terminal and ald $D_{P p}$ with a His-tag on N-terminal, respectively. The aldH gene, with a Histag on N-terminal, was amplified by PCR using E. coli MG1655 genomic DNA as a template and cloned into the p59 plasmid by replacing the ald $D_{H b}$ gene. This plasmid was labeled as p99. The $d h a T_{K p}$ gene with a His-tag on C-terminal or puuC gene with a His-tag on $\mathrm{N}$-terminal was amplified by PCR using $K$. pneumoniae DSMZ 2026 genomic DNA as a template and cloned into the p59 plasmid by replacing the $a d h P$ or ald $D_{H b}$ gene. These plasmids were labeled as p95 and p100. All the recombinant plasmids were conjugated into $H$. bluephagenesis TD $\Delta d d d A$ and cultivated in a defined minimal medium containing $20 \mathrm{~g} \mathrm{~L}^{-1}$ glucose, $20 \mathrm{~g} \mathrm{~L}^{-1}$ 1,3-propanediol, and $3 \mathrm{~g} \mathrm{~L}^{-1}$ acetic acid. The recombinant strains were cultured in conical flasks and grown for $24 \mathrm{~h}$. Subsequently, they were harvested by centrifugation under $4000 \times g$ and $4{ }^{\circ} \mathrm{C}$ for 20 min. Proteins were purified by a His-tag Protein Purification Kit (Beyotime, China) and stored at $-80{ }^{\circ} \mathrm{C}$ for further usage. The enzyme activity was assayed by a VarioskanFlash plate reader (Version 4.00.53, Thermo Scientific, USA).

The reductive activity (forward reaction) of AdhP, DhaT $\mathrm{T}_{\mathrm{Pp}}$, and $\mathrm{DhaT}_{\mathrm{Kp}}$ were assayed as follows, respectively: First, the reaction mixture containing $50 \mathrm{mM}$ Tris$\mathrm{HCl}$ buffer ( $\mathrm{pH} 7.0$ ) and $10 \mathrm{nM}$ enzyme was incubated for $2 \mathrm{~min}$ at $37^{\circ} \mathrm{C}$. The reaction was initiated by the addition of $0.1 \mathrm{mM}$ of NADH and $5 \mathrm{mM} 3 \mathrm{HPA}$. The enzyme activity was determined by measuring the NADH oxidation using the molar extinction coefficient $\left(\Delta \varepsilon_{340}\right)$ of $6.22 \times 10^{3} \mathrm{M}^{-1} \mathrm{~cm}^{-1}$. The oxidative activity (reverse reaction) of AdhP, DhaT $\mathrm{T}_{\mathrm{Pp}}$, and $\mathrm{DhaT}_{\mathrm{Kp}}$ were measured as follows: The reaction mixture containing $100 \mathrm{mM}$ potassium carbonate buffer ( $\mathrm{pH} 9.0), 30 \mathrm{mM}$ ammonium sulfate, and $100 \mathrm{nM}$ enzyme was incubated for $2 \mathrm{~min}$ at $37^{\circ} \mathrm{C}$. The reaction was initiated by the addition of $2 \mathrm{mM}$ of $\mathrm{NAD}^{+}$and $150 \mathrm{mM} \mathrm{1,3-}$ propanediol ${ }^{51}$. The activity of the aldehyde dehydrogenase was determined by measuring the level of $\mathrm{NAD}^{+}$reduction to $\mathrm{NADH}$ at $340 \mathrm{~nm}$. The reaction mixture containing $100 \mathrm{mM}$ potassium phosphate buffer ( $\mathrm{pH} 8.0), 1 \mathrm{mM}$ DTT, and $100 \mathrm{nM}$ enzyme was incubated at $37^{\circ} \mathrm{C}$ for $5 \mathrm{~min}$. The reaction was initiated by adding $2.0 \mathrm{mM} 3 \mathrm{HPA}$ and $2.0 \mathrm{mM} \mathrm{NAD}^{+}$. The amount of NADH formed was studied using a molar extinction coefficient $(\Delta \varepsilon 340)$ of $6.22 \times 10^{3} \mathrm{M}^{-1} \mathrm{~cm}^{-1}$. One unit of aldehyde dehydrogenase activity was defined as the amount of enzyme needed to reduce $1 \mu \mathrm{mol}$ of $\mathrm{NAD}^{+}$to $\mathrm{NADH}$ in $1 \mathrm{~min}^{49}$.

NADH assays. All cells were harvested after fermentation via centrifugation under $2500 \times g$ at $4{ }^{\circ} \mathrm{C}$ for $10 \mathrm{~min} .200 \mu \mathrm{L}$ cells were collected from each sample, followed by washing using ice-cold PBS at $\mathrm{pH}$ 7.4. The obtained pellet was disrupted by a chemical lysis method using $400 \mu \mathrm{L}$ BugBuster ${ }^{\circ}$ Master Mix (ThermoFisher Scientific Inc., USA). All operations were strictly conducted based on instructions of NAD/NADH Assay Kit S0175 (Beyotime, China).

3HP and PHB production in a $\mathbf{7} \mathbf{L}$ fermenter. Fed-batch studies were performed in a $7 \mathrm{~L}$ BioFlo fermenter (New Brunswick Scientific, U.S.A) with a $3 \mathrm{~L}$ working volume without sterilization. The growth temperature was $37^{\circ} \mathrm{C}$, maintained via a cooling circulation pump (Henan Jinghua Instrument, China). The $\mathrm{pH}$ was maintained at 8.5 using an automatic pump added $5 \mathrm{M} \mathrm{NaOH}$ into the culture system when needed. The fermentation agitation speed was set at $200 \mathrm{rpm}$ at the 
beginning, coupled with dissolved oxygen (DO) concentration, and gradually increased to $800 \mathrm{rpm}$ to maintain the DO level above $30 \%$ compared with the one in a cell-free fermenter (set as $100 \%$ ). The agitation speed was maintained at 800 rpm to the end of fermentation. The inlet airflow rate was maintained at $3 \mathrm{~L} \mathrm{~min}^{-1}$ during the entire fermentation process.

The seed culture has the same medium as that in shake flasks. A $300 \mathrm{~mL}$ seed culture ( $10 \%$ of the total volume) was prepared for inoculation into the fermenter. The $\mathrm{OD}_{600}$ of the seeds was adjusted to 3 . For fermentor studies, low-cost substrates were used and the defined minimal medium was modified by utilizing $16 \mathrm{~g} \mathrm{~L}^{-1}$ corn steep (Baisheng, Shandong Biotechnology Co., Ltd., China) instead of the expensive yeast extract, the $\mathrm{NaCl}$ concentration was reduced to 40 from $60 \mathrm{~g} \mathrm{~L}^{-1}$.

For $3 \mathrm{HP}$ production, a two-stage and intermittent feeding strategy were adopted using different feeding solutions. $400 \mathrm{~mL}$ feeding solution I containing $800 \mathrm{~g} \mathrm{~L}^{-1}$ glucose and $30 \mathrm{~g} \mathrm{~L}^{-1}$ urea was used to increase cell mass accumulation and PHB formation during the first $16 \mathrm{~h}$. $200 \mathrm{~mL}$ feeding Solution II containing $800 \mathrm{~g} \mathrm{~L}^{-1}$ glucose and $15 \mathrm{~g} \mathrm{~L}^{-1}$ urea was added after feeding Solution I exhausted. The flow rates of the feeding solutions depended on the residual glucose concentrations that should be controlled between 5 and $10 \mathrm{~g} \mathrm{~L}^{-1}$ measured using the GA-3 blood glucose meter and GA-3 blood glucose test strip (Sinocare, China). $60 \mathrm{~g} \mathrm{~L}^{-1} 1,3$-propanediol and $6 \mathrm{~g} \mathrm{~L}^{-1}$ acetic acid were added three times separately at 24,32 , and $38 \mathrm{~h}$ after the beginning of the fermentations. $\mathrm{OD}_{600}$ of the cultured bacteria was measured by a V-5600 visible light spectrophotometer (Shanghai Yuanxi Instrument, China).

For $\mathrm{P} 3 \mathrm{HB} 3 \mathrm{HP}$ production, a two-stage feeding strategy was used with different feeding solutions. $400 \mathrm{~mL}$ feeding solution I containing $800 \mathrm{~g} \mathrm{~L}^{-1}$ glucose, $30 \mathrm{~g} \mathrm{~L}^{-1}$ urea, and $75 \mathrm{~g} \mathrm{~L}^{-1} 1,3$-propanediol was used to increase cell mass and P3HB3HP formation during the first $20 \mathrm{~h} .200 \mathrm{~mL}$ feeding solution II containing $800 \mathrm{~g} \mathrm{~L}^{-1}$ glucose, $15 \mathrm{~g} \mathrm{~L}^{-1}$ urea, and $75 \mathrm{~g} \mathrm{~L}^{-1} 1,3$-propanediol was added after feeding solution I exhausted.

PHA purification and analysis. After shake-flask growth, cells were harvested by centrifugation (CR21 GIII, Hitachi, Japan) at $8000 \times g$ for $10 \mathrm{~min}$, washed twice with distilled water, and placed in $-80^{\circ} \mathrm{C}$ for $4 \mathrm{~h}$. After cells lyophilizing for $12 \mathrm{~h}$, their intracellular PHA was extracted using a Soxtec 2050 Soxhlet extractor (Foss, Denmark). PHA polymers were purified by re-dissolving the extracted materials in chloroform, then precipitated using absolute ethanol, centrifuged at $12,000 \times g$ for 10 min to harvest the purified PHA materials, followed by lyophilization again to remove residual ethanol.

The dry cell mass was determined by weighing $30 \mathrm{~mL}$ lyophilized centrifuged bacterial cells. For the esterification reaction, 40-60 mg lyophilized cells were placed into a $2 \mathrm{~mL}$ esterification reagent containing $3 \%$ sulfuric acid and $0.1 \%$ benzoic acid, both dissolved in absolute methanol. After adding $2 \mathrm{~mL}$ chloroform, the whole reaction was performed at $100^{\circ} \mathrm{C}$ for $4 \mathrm{~h}$. PHA contents were determined by GC-2014 gas chromatography (Shimadzu, Japan). P3HB (99.9\%, Sigma-Aldrich, Germany) was used as standards, respectively. The peak of benzoic acid was regarded as the internal standard.

\section{Chemical quantification using high-performance liquid chromatography}

Concentrations of $3 \mathrm{HP}$, glucose, acetic acid, and 1,3-propanediol were determined via high-performance liquid chromatography (HPLC). An LC-20 instrument (Shimadzu, Japan) with an Aminex HPX-87H column (Bio-Rad, U.S.A) and a RID-10A refractive index detector (Shimadzu) was employed. The mobile phase was a $5 \mathrm{mM}$ degassed $\mathrm{H}_{2} \mathrm{SO}_{4}$ with a flow rate of $0.5 \mathrm{~mL} \mathrm{~min}^{-1}$. The column temperature was maintained at $55^{\circ} \mathrm{C}^{70}$. The culture media were centrifuged at $12,000 \times g$ and $4{ }^{\circ} \mathrm{C}$ for 2 min to obtain the supernatant, which was then filtered through a $0.22 \mu \mathrm{m}$ polyethersulfone membrane syringe filter (Jinglong, China) and used as the samples with a loading volume of $30 \mu \mathrm{L}$. Standards of the above chemicals were prepared by five different concentrations to draw the standard curves, from which the concentrations of the sample were calculated.

Nuclear magnetic resonance. PHA samples $(2-5 \mathrm{mg})$ were dissolved in deuter ated chloroform for ${ }^{1} \mathrm{H}$ NMR with a JNM-ECA600 nuclear magnetic resonance instrument (JEOL, Japan). Chemical shifts were given in ppm while $0.03 \% \mathrm{v} / \mathrm{v}$ tetramethylsilane (TMS) served as an internal standard to calibrate against the signal of samples.

Reporting summary. Further information on research design is available in the Nature Research Reporting Summary linked to this article.

\section{Data availability}

RNA-seq data that support the findings of this study have been deposited in the Gene

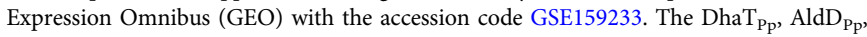
DddA, AldD $\mathrm{Hb}_{\mathrm{Hb}}$ and $\mathrm{AdhP}$ sequences used in this study are under the accession numbers AAN68411.1, AAN66172.1, WP_009721523.1, WP_009721344.1 and WP_039868491.1, respectively. Data supporting the findings of this work are available within the paper and its supplementary information files. A reporting summary for this article is available as a supplementary information file. All relevant data are available from the authors upon reasonable request. Source data are provided with this paper.
Received: 16 June 2020; Accepted: 2 February 2021;

Published online: 08 March 2021

\section{References}

1. Chen, Y., Bao, J., Kim, I.-K., Siewers, V. \& Nielsen, J. Coupled incremental precursor and co-factor supply improves 3-hydroxypropionic acid production in Saccharomyces cerevisiae. Metab. Eng. 22, 104-109 (2014).

2. Borodina, I. et al. Establishing a synthetic pathway for high-level production of 3-hydroxypropionic acid in Saccharomyces cerevisiae via $\beta$-alanine. Metab. Eng. 27, 57-64 (2015).

3. Choi, S., Song, C. W., Shin, J. H. \& Lee, S. Y. Biorefineries for the production of top building block chemicals and their derivatives. Metab. Eng. 28, 223-239 (2015)

4. Karp, E. M. et al. Renewable acrylonitrile production. Science 358, 1307-1310 (2017).

5. Della Pina, C., Falletta, E. \& Rossi, M. A green approach to chemical building blocks. case 3-hydroxypropanoic acid. Green. Chem. 13, 1624-1632 (2011).

6. Kumar, V., Ashok, S. \& Park, S. Recent advances in biological production of 3 hydroxypropionic acid. Biotechnol. Adv. 31, 945-961 (2013).

7. Vollenweider, S. \& Lacroix, C. 3-Hydroxypropionaldehyde: applications and perspectives of biotechnological production. Appl. Microbiol. Biotechnol. 64, 16-27 (2004)

8. Lan, E. I. et al. Metabolic engineering of cyanobacteria for photosynthetic 3hydroxypropionic acid production from $\mathrm{CO} 2$ using Synechococcus elongatus PCC 7942. Metab. Eng. 31, 163-170 (2015).

9. Liao, J. C., Mi, L., Pontrelli, S. \& Luo, S. Fuelling the future: microbial engineering for the production of sustainable biofuels. Nat. Rev. Microbiol. 14, 288 (2016).

10. Liu, C. et al. Functional balance between enzymes in malonyl-CoA pathway for 3-hydroxypropionate biosynthesis. Metab. Eng. 34, 104-111 (2016).

11. Song, C. W., Kim, J. W., Cho, I. J. \& Lee, S. Y. Metabolic engineering of Escherichia coli for the production of 3-hydroxypropionic acid and malonic acid through $\beta$-alanine route. ACS Synth. Biol. 5, 1256-1263 (2016).

12. Yang, Y., Lin, Y., Li, L., Linhardt, R. J. \& Yan, Y. Regulating malonyl-CoA metabolism via synthetic antisense RNAs for enhanced biosynthesis of natural products. Metab. Eng. 29, 217-226 (2015).

13. de Fouchécour, F., Sánchez-Castañeda, A.-K., Saulou-Bérion, C. \& Spinnler, H. E. Process engineering for microbial production of 3-hydroxypropionic acid. Biotechnol. Adv. 36, 1207-1222 (2018).

14. Chen, Z. \& Liu, D. Toward glycerol biorefinery: metabolic engineering for the production of biofuels and chemicals from glycerol. Biotechnol. Biofuels 9, 205 (2016).

15. Wang, J. et al. Rational engineering of diol dehydratase enables 1, 4-butanediol biosynthesis from xylose. Metab. Eng. 40, 148-156 (2017).

16. Chu, H. S. et al. Metabolic engineering of 3-hydroxypropionic acid biosynthesis in Escherichia coli. Biotechnol. Bioeng. 112, 356-364 (2015).

17. Chen, Z. et al. Metabolic engineering of Corynebacterium glutamicum for the production of 3-hydroxypropionic acid from glucose and xylose. Metab. Eng. 39, 151-158 (2017).

18. Li, Y., Wang, X., Ge, X. \& Tian, P. High production of 3-hydroxypropionic acid in Klebsiella pneumoniae by systematic optimization of glycerol metabolism. Sci. Rep. 6, 26932 (2016).

19. Zhao, P., Ren, M., Ge, X., Tian, P. \& Tan, T. Development of orthogonal T7 expression system in Klebsiella pneumoniae. Biotechnol. Bioeng. 117, 2446-2459 (2020)

20. Li, X., Chen, L., Wang, X. \& Tian, P. Physiological investigations of the influences of byproduct pathways on 3-hydroxypropionic acid production in Klebsiella pneumoniae. J. Basic Microbiol. 59, 1195-1207 (2019).

21. Ashok, S. et al. Production of 3-hydroxypropionic acid from glycerol by recombinant Klebsiella pneumoniae $\Delta \mathrm{dhaT} \Delta \mathrm{yqhD}$ which can produce vitamin B12 naturally. Biotechnol. Bioeng. 110, 511-524 (2013).

22. Ashok, S. et al. Effect of puuC overexpression and nitrate addition on glycerol metabolism and anaerobic 3-hydroxypropionic acid production in recombinant Klebsiella pneumoniae $\Delta \mathrm{glpK} \Delta \mathrm{dhaT}$. Metab. Eng. 15, 10-24 (2013).

23. Kumar, V. et al. Simultaneous production of 3-hydroxypropionic acid and 1, 3-propanediol from glycerol using resting cells of the lactate dehydrogenasedeficient recombinant Klebsiella pneumoniae overexpressing an aldehyde dehydrogenase. Bioresour. Technol. 135, 555-563 (2013).

24. Xu, X. L., Zhang, G. L., Wang, L. W., Ma, B. B. \& Li, C. Quantitative analysis on inactivation and reactivation of recombinant glycerol dehydratase from Klebsiella pneumoniae XJPD-Li. J. Mol. Catal. B 56, 108-114 (2009).

25. Saxena, R., Anand, P., Saran, S. \& Isar, J. Microbial production of 1, 3propanediol: recent developments and emerging opportunities. Biotechnol. Adv. 27, 895-913 (2009). 
26. Zhao, L., Lin, J., Wang, H., Xie, J. \& Wei, D. Development of a two-step process for production of 3-hydroxypropionic acid from glycerol using Klebsiella pneumoniae and Gluconobacter oxydans. Bioprocess Biosyst. Eng. 38, 2487-2495 (2015).

27. Zhu, J. et al. Identification of the enzymes responsible for 3-hydroxypropionic acid formation and their use in improving 3-hydroxypropionic acid production in Gluconobacter oxydans DSM 2003. Bioresour. Technol. 265, 328-333 (2018).

28. Li, J. et al. Immobilization of Acetobacter sp. CGMCC 8142 for efficient biocatalysis of 1, 3-propanediol to 3-hydroxypropionic acid. Biotechnol. Bioproc. E 21, 523-530 (2016).

29. Chen, G. Q. \& Jiang, X. R. Next generation industrial biotechnology based on extremophilic bacteria. Curr. Opin. Biotechnol. 50, 94-100 (2018).

30. Dan, T., Xue, Y. S., Aibaidula, G. \& Chen, G. Q. Unsterile and continuous production of polyhydroxybutyrate by Halomonas TD01. Bioresour. Technol. 102, 8130-8136 (2011).

31. Fu, X. Z. et al. Development of Halomonas TD01 as a host for open production of chemicals. Metab. Eng. 23, 78-91 (2014).

32. Chen, X. et al. Engineering Halomonas bluephagenesis TD01 for non-sterile production of poly (3-hydroxybutyrate-co-4-hydroxybutyrate). Bioresour. Technol. 244, 534-541 (2017).

33. Qin, Q. et al. CRISPR/Cas9 editing genome of extremophile Halomonas spp. Metab. Eng. 47, 219-229 (2018).

34. Zhao, H. et al. Novel T7-like expression systems used for Halomonas. Metab. Eng. 39, 128-140 (2017).

35. Thorwall, S., Schwartz, C., Chartron, J. W. \& Wheeldon, I. Stress-tolerant nonconventional microbes enable next-generation chemical biosynthesis. Nat. Chem. Biol. 16, 113-121 (2020).

36. Nikel, P. I., Chavarria, M., Danchin, A. \& de Lorenzo, V. From dirt to industrial applications: Pseudomonas putida as a synthetic biology chassis for hosting harsh biochemical reactions. Curr. Opin. Chem. Biol. 34, 20-29 (2016).

37. Tan, D., Wu, Q., Chen, J. C. \& Chen, G. Q. Engineering Halomonas TD01 for the low-cost production of polyhydroxyalkanoates. Metab. Eng. 26, 34-47 (2014).

38. Ye, J. et al. Engineering of Halomonas bluephagenesis for low cost production of poly (3-hydroxybutyrate-co-4-hydroxybutyrate) from glucose. Metab. Eng. 47, 143-152 (2018)

39. Chen, Y. et al. Chromosome engineering of the TCA cycle in Halomonas bluephagenesis for production of copolymers of 3-hydroxybutyrate and 3hydroxyvalerate (PHBV). Metab. Eng. 54, 69-82 (2019).

40. Yu, L. P. et al. Biosynthesis of functional polyhydroxyalkanoates by engineered Halomonas bluephagenesis. Metab. Eng. 59, 119-130 (2020).

41. Zhang, X., Lin, Y. \& Chen, G. Q. Halophiles as chassis for bioproduction. Adv. Biosyst. 2, 1800088 (2018).

42. Vreeland, R. H., Litchfield, C., Martin, E. \& Elliot, E. Halomonas elongata, a new genus and species of extremely salt-tolerant bacteria. Int. J. Syst. Evol. Microbiol. 30, 485-495 (1980).

43. Warnecke, T. \& Gill, R. T. Organic acid toxicity, tolerance, and production in Escherichia coli biorefining applications. Microb. Cell. Fact. 4, 25 (2005).

44. Todd, J. D. et al. Molecular dissection of bacterial acrylate catabolism-unexpected links with dimethylsulfoniopropionate catabolism and dimethyl sulfide production. Environ. Microbiol. 12, 327-343 (2010).

45. Zhou, S., Ainala, S. K., Seol, E., Nguyen, T. T. \& Park, S. Inducible gene expression system by 3-hydroxypropionic acid. Biotechnol. Biofuels. 8, 169 (2015).

46. Seok, J. Y. et al. Directed evolution of the 3-hydroxypropionic acid production pathway by engineering aldehyde dehydrogenase using a synthetic selection device. Metab. Eng. 47, 113-120 (2018).

47. Zhou, S., Ashok, S., Ko, Y., Kim, D.-M. \& Park, S. Development of a deletion mutant of Pseudomonas denitrificans that does not degrade 3hydroxypropionic acid. Appl. Microbiol. Biotechnol. 98, 4389-4398 (2014).

48. $\mathrm{Li}, \mathrm{T}$. et al. Engineering of core promoter regions enables the construction of constitutive and inducible promoters in Halomonas sp. Biotechnol. J. 11, 219-227 (2016).

49. Ko, Y., Ashok, S., Zhou, S., Kumar, V. \& Park, S. Aldehyde dehydrogenase activity is important to the production of 3-hydroxypropionic acid from glycerol by recombinant Klebsiella pneumoniae. Process. Biochem. 47, 1135-1143 (2012).

50. Martínez-García, E., Benedetti, I., Hueso, A. \& de Lorenzo, V. Mining environmental plasmids for synthetic biology parts and devices. Plasmids $\mathbf{3}$, 633-649 (2015).

51. Lama, S. et al. Characterization of 1, 3-propanediol oxidoreductase (DhaT) from Klebsiella pneumoniae J2B. Biotechnol. Bioproc. E 20, 971-979 (2015).

52. Raj, S. M., Rathnasingh, C., Jung, W. C., Selvakumar, E. \& Park, S. A novel $\mathrm{NAD}+$-dependent aldehyde dehydrogenase encoded by the puuC gene of
Klebsiella pneumoniae DSM 2026 that utilizes 3-hydroxypropionaldehyde as a substrate. Biotechnol. Bioproc. E 15, 131-138 (2010).

53. Stäubli, A. \& Boelsterli, U. A. The labile iron pool in hepatocytes: prooxidantinduced increase in free iron precedes oxidative cell injury. Am. J. Physiol. 274, G1031-G1037 (1998)

54. Wei, N., Quarterman, J., Kim, S. R., Cate, J. H. \& Jin, Y.-S. Enhanced biofuel production through coupled acetic acid and xylose consumption by engineered yeast. Nat. Commun. 4, 1-8 (2013).

55. Chen, L. et al. Engineering NADH/NAD + ratio in Halomonas bluephagenesis for enhanced production of polyhydroxyalkanoates (PHA). Metab. Eng. 49, 275-286 (2018)

56. Andreessen, B., Taylor, N. \& Steinbuchel, A. Poly(3-Hydroxypropionate): a promising alternative to fossil fuel-based materials. Appl. Environ. Microbiol. 80, 6574-6582 (2014).

57. Meng, D. C. et al. Production and characterization of poly(3hydroxypropionate-co-4-hydroxybutyrate) with fully controllable structures by recombinant Escherichia coli containing an engineered pathway. Metab. Eng. 14, 317-324 (2012).

58. Meng, D. C. et al. Production of poly(3-hydroxypropionate) and poly(3hydroxybutyrate-co-3-hydroxypropionate) from glucose by engineering Escherichia coli. Metab. Eng. 29, 189-195 (2015).

59. Wang, Q. et al. Production of block copolymer poly (3-hydroxybutyrate)block-poly (3-hydroxypropionate) with adjustable structure from an inexpensive carbon source. ACS Macro Lett. 2, 996-1000 (2013)

60. Prieto, A. et al. A holistic view of polyhydroxyalkanoate metabolism in Pseudomonas putida. Environ. Microbiol. 18, 341-357 (2016).

61. Naseri, G. \& Koffas, M. A. Application of combinatorial optimization strategies in synthetic biology. Nat. Commun. 11, 1-14 (2020).

62. $\mathrm{Du}, \mathrm{H}$. et al. Engineering Halomonas bluephagenesis for L-threonine production. Metab. Eng. 47, 219-229 (2020).

63. Zhang, J. et al. Substrate profiling and tolerance testing of Halomaons TD01 suggest its potential application in sustainable manufacturing of chemicals. J. Biotechnol. 316, 1-5 (2020).

64. Warnecke, T., Lynch, M., Karimpour-Fard, A., Sandoval, N. \& Gill, R. A genomics approach to improve the analysis and design of strain selections. Metab. Eng. 10, 154-165 (2008).

65. Bertsch, J., Siemund, A. L., Kremp, F. \& Müller, V. A novel route for ethanol oxidation in the acetogenic bacterium Acetobacterium woodii: the acetaldehyde/ethanol dehydrogenase pathway. Environ. Microbiol. 18, 2913-2922 (2016)

66. Zhou, Q. et al. Production of 3-hydroxypropionate homopolymer and poly (3 hydroxypropionate-co-4-hydroxybutyrate) copolymer by recombinant Escherichia coli. Metab. Eng. 13, 777-785 (2011).

67. Choi, S. Y. et al. One-step fermentative production of poly (lactate-coglycolate) from carbohydrates in Escherichia coli. Nat. Biotechnol. 34, 435 (2016).

68. Tian, P. et al. Fundamental CRISPR-Cas9 tools and current applications in microbial systems. Synth. Syst. Biotechnol. 2, 219-225 (2017).

69. Schmittgen, T. D. \& Livak, K. J. Analyzing real-time PCR data by the comparative C T method. Nat. Protoc. 3, 1101 (2008).

70. Tokuyama, K. et al. Magnesium starvation improves production of malonylCoA-derived metabolites in Escherichia coli. Metab. Eng. 52, 215-223 (2019)

\section{Acknowledgements}

This research was financially supported by a grant from the Ministry of Sciences and Technology (Grant No. 2018YFA0900200) to G.-Q.C., grants from the National Natural Science Foundation of China (Grant No. 21761132013, 31870859, 31900045), National Postdoctoral Program for Innovative Talents (Grant No. BX201700130) and China Postdoctoral Science Foundation (Grant No. 2018M640116) contributed to this research. The pSEVA plasmids were gifts from Prof. Victor de Lorenzo of CSIC, Spain. This project is also funded by the National Natural Science Foundation of China (Grant Numbers 31961133017, 31961133018, 31961133019). These grants are part of MIX-UP, a joint NSFC and EU H2020 collaboration. In Europe, MIX-UP has received funding from the European Union's Horizon 2020 research and innovation program under grant Agreement No. 870294.

\section{Author contributions}

X.-R.J. designed and performed the study. X.-R.J. and X.Y. performed experiments and conducted the data analysis. L.-P.Y. assisted with NMR analysis. X.-Y.L. assisted with fermentation. X.-R.J., X.Y., and X.-Y.L. wrote the manuscript. G.-Q.C. supervised the project and helped prepare the manuscript.

\section{Competing interests}

The authors declare no competing interests. 


\section{Additional information}

Supplementary information The online version contains supplementary material available at https://doi.org/10.1038/s41467-021-21632-3.

Correspondence and requests for materials should be addressed to G.-Q.C.

Peer review information Nature Communications thanks Tae Hyeon Yoo, Sunghoon Park and the other, anonymous, reviewer(s) for their contribution to the peer review of this work.

Reprints and permission information is available at http://www.nature.com/reprints

Publisher's note Springer Nature remains neutral with regard to jurisdictional claims in published maps and institutional affiliations. (c) (1) Open Access This article is licensed under a Creative Commons Attribution 4.0 International License, which permits use, sharing, adaptation, distribution and reproduction in any medium or format, as long as you give appropriate credit to the original author(s) and the source, provide a link to the Creative Commons license, and indicate if changes were made. The images or other third party material in this article are included in the article's Creative Commons license, unless indicated otherwise in a credit line to the material. If material is not included in the article's Creative Commons license and your intended use is not permitted by statutory regulation or exceeds the permitted use, you will need to obtain permission directly from the copyright holder. To view a copy of this license, visit http://creativecommons.org/ licenses/by/4.0/.

(C) The Author(s) 2021 